\title{
ON THE ENERGY OF BOUND STATES FOR MAGNETIC SCHRÖDINGER OPERATORS
}

\author{
SØREN FOURNAIS AND AYMAN KACHMAR
}

\begin{abstract}
We provide a leading order semiclassical asymptotics of the energy of bound states for magnetic Neumann Schrödinger operators in two dimensional (exterior) domains with smooth boundaries. The asymptotics is valid all the way up to the bottom of the essential spectrum. When the spectral parameter is varied near the value where bound states become allowed in the interior of the domain, we show that the energy has a boundary and a bulk component. The estimates rely on coherent states, in particular on the construction of 'boundary coherent states', and magnetic Lieb-Thirring estimates.
\end{abstract}

\section{INTRODUCTION}

Let $\Omega^{\prime} \subset \mathbb{R}^{2}$ be an open and bounded domain with regular boundary. We will consider both the case of interior domains $\Omega=\Omega^{\prime}$ and exterior domains $\Omega=\mathbb{R}^{2} \backslash \Omega^{\prime}$.

Consider the magnetic Schrödinger operator, or magnetic Laplacian, in $\Omega$. It has been observed by many authors (see for example [12]) that the presence of Neumann boundary conditions has an effect similar to that of a negative electric potential (also Robin boundary conditions have a similar effect, see [13, 14]). For the case of the present discussion, consider a constant magnetic field, more generally we will impose the hypothesis (1.6) below. One aspect of the analogy is that the Neumann boundary condition leads to a discrete spectrum below the lowest Landau level (in the case of an exterior domain, the (magnetic) Laplacian may have essential spectrum). It is that spectrum which we will discuss in the present paper.

One motivation for our work is the analysis of type II superconductivity for applied magnetic fields close to the second critical field (see [17, Problem 2.2 .8$, p. 491] for a discussion of this point). In the setting of superconductivity, one encounters the asymptotic regime of a large magnetic field, but this is equivalent (through a simple change of parameter) to the semiclassical regime considered in this paper.

Many different investigations of the discrete spectrum close to the lowest Landau level have appeared (see [10, 19, 20] and references therein). Of particular importance for our present investigation is the recent paper [9]. In that paper the counting function is studied up to an energy strictly below the lowest Landau level. Inspired by work on the magnetic problems without boundaries - but with electric potential - see [16, we shift focus from the counting function to the energy, i.e. the sum of the eigenvalues. This allows us to obtain 'semiclassical' results all the way up to the lowest Landau level

Date: November 10, 2018. 
(Theorem 1.1 below). Furthermore, by varying slightly the energy up to which we sum the eigenvalues, we can demonstrate how the bulk or interior bound states start to contribute to the leading order asymptotics for the energy (Theorem 1.4 below).

Let us now move to the precise statement of our results. We consider the (Neumann) Schrödinger operator with magnetic field :

$$
P_{h, \Omega}=-(h \nabla-i \mathbf{A})^{2}=(-i h \nabla-\mathbf{A})^{2},
$$

whose domain is,

$$
\begin{aligned}
D\left(P_{h, \Omega}\right)=\left\{u \in L^{2}(\Omega):\right. & (h \nabla-i \mathbf{A})^{j} u \in L^{2}(\Omega), j=1,2, \\
& \nu \cdot(h \nabla-i \mathbf{A}) u=0 \text { on } \partial \Omega\} .
\end{aligned}
$$

Here $h>0$ is a small parameter (the so called semi-classical parameter), $\nu$ is the unit outward normal vector of the boundary $\partial \Omega$ and $\mathbf{A} \in C^{2}\left(\bar{\Omega} ; \mathbb{R}^{2}\right)$ is a given vector field - the vector potential. The magnetic field is a function and is given by

$$
B=\operatorname{curl} \mathbf{A}=\partial_{x_{1}} A_{2}-\partial_{x_{2}} A_{1} .
$$

With this magnetic field we associate the quantities

$$
b=\inf _{x \in \bar{\Omega}} B(x), \quad b^{\prime}:=\inf _{x \in \partial \Omega} B(x) .
$$

Assuming that $b>0$, we know that the following leading order asymptotic expansion holds for the bottom of the spectrum of $P_{h, \Omega}$ (see for example [12])

$$
\inf \operatorname{Spec} P_{h, \Omega}=h \min \left(b, \Theta_{0} b^{\prime}\right)+o(h) .
$$

Here $\left.\Theta_{0} \in\right] 0,1[$ is a universal constant (the definition will be recalled in (2.13) below).

We shall assume that the magnetic field is bounded, positive and satisfies

$$
b>\Theta_{0} b^{\prime}>0 .
$$

Notice that the hypothesis (1.6) is satisfied in the case of a constant magnetic field. Under the hypothesis (1.6) eigenvalues strictly below $b h$ are associated with eigenfunctions localized near the boundary. One of the objectives of the present paper is to prove that - for the energy - this remains true all the way up to the value $b h$.

In order to state our main results, we need to recall some facts concerning the harmonic oscillator on the semi-axis $\mathbb{R}_{+}$. For $\xi \in \mathbb{R}$, we denote by $\mu_{1}(\xi)$ the lowest eigenvalue of the operator

$$
-\partial_{t}^{2}+(t-\xi)^{2} \quad \text { in } \quad L^{2}\left(\mathbb{R}_{+}\right)
$$

with standard Neumann boundary condition at the origin. It is well known (see [3, 4]) that the function $\xi \mapsto \mu_{1}(\xi)$ is smooth,

$$
\mu_{1}(\xi)<1, \text { for all } \xi \in \mathbb{R}_{+}, \quad \mu_{1}(\xi)>1, \text { for all } \xi \in \mathbb{R}_{-},
$$

and the integral

$$
\int_{0}^{\infty}\left(\mu_{1}(\xi)-1\right) d \xi=-\int_{\mathbb{R}}\left[\mu_{1}(\xi)-1\right]_{-} d \xi
$$


is negative and finite. Here we introduced the notation $[x]_{-}$, more generally, we will use the following positive functions

$$
[x]_{+}=\left\{\begin{array}{ll}
x, & x \geq 0, \\
0, & x<0,
\end{array} \quad[x]_{-}= \begin{cases}0, & x \geq 0 \\
-x, & x<0\end{cases}\right.
$$

Our result is the following.

Theorem 1.1. Suppose $\Omega$ is either an exterior or an interior domain. Suppose $B$ satisfies (1.6). Then the spectrum of $P_{h, \Omega}$ below bh is discrete,

$$
\left.\sigma\left(P_{h, \Omega}\right) \cap\right]-\infty, b h\left[=\left\{e_{1}(h), e_{2}(h), \ldots\right\},\right.
$$

and the sequence $\left\{e_{j}(h)-b h\right\}_{j \geq 1}$ is summable,

$$
\sum_{j}\left[e_{j}(h)-b h\right]_{-}=-\operatorname{tr}\left(\left(P_{h, \Omega}-b h\right) \mathbf{1}_{-\infty, b h[}\left(P_{h, \Omega}\right)\right) \quad \text { is finite. }
$$

Moreover, the following asymptotic formula holds,

$$
\begin{aligned}
\lim _{h \rightarrow 0} h^{-1 / 2} \sum_{j}\left[e_{j}(h)-b h\right]_{-} & \\
= & \frac{1}{2 \pi} \int_{\partial \Omega} \int_{-\infty}^{\infty} B(x)^{3 / 2}\left[\frac{b}{B(x)}-\mu_{1}(\xi)\right]_{+} d \xi d s(x) .
\end{aligned}
$$

Here $d s(x)$ denotes integration with respect to arc-length along the boundary $\partial \Omega$.

Remark 1.2. Assuming that the magnetic field $B=\operatorname{curl} \mathbf{A}$ is constant, $B(x)=b$, the asymptotic formula of Theorem 1.1 reads

$$
\lim _{h \rightarrow 0} h^{-1 / 2}\left(\sum_{j}\left[e_{j}(h)-b h\right]_{-}\right)=\frac{|\partial \Omega| b^{3 / 2}}{2 \pi} \int_{0}^{\infty}\left(1-\mu_{1}(\xi)\right) d \xi .
$$

Remark 1.3. In [9], an asymptotic formula is obtained for the number $N(\lambda h)$ of eigenvalues of $P_{h, \Omega}$ below $\lambda h$, for a given $\lambda<b$. The precise result is the following :

$$
\lim _{h \rightarrow 0} h^{1 / 2} N(\lambda h)=\frac{1}{2 \pi} \int_{\left\{(x, \xi) \in \partial \Omega \times \mathbb{R}: B(x) \mu_{1}(\xi)<\lambda\right\}} B(x)^{1 / 2} d \xi d s(x) .
$$

Integration of (1.9) yields the following formula for the energy,

$$
\begin{aligned}
\lim _{h \rightarrow 0} h^{-1 / 2}\left(\sum_{j}\right. & {\left.\left[e_{j}(h)-\lambda h\right]_{-}\right) } \\
= & \frac{1}{2 \pi} \int_{\partial \Omega} \int_{0}^{\infty} B(x)^{3 / 2}\left[\frac{\lambda}{B(x)}-\mu_{1}(\xi)\right]_{+} d \xi d s(x) .
\end{aligned}
$$

However, the proof we give to Theorem 1.1 gives equally (1.10) (we only give the details for the harder case $\lambda=b$ ), and hence, by differentiating (1.10), (more precisely, the 'differentiation' needed is the technique used to go from energies to densities in semiclassical problems, see [6] for details) we provide an alternative proof of (1.9). 
In the next theorem, we restrict to the case of interior domains, i.e. bounded $\Omega$. In this specific case, the operator $P_{h, \Omega}$ has compact resolvent and hence its spectrum is purely discrete. Let us denote by $\left\{e_{j}(h)\right\}$ the increasing sequence of eigenvalues of $P_{h, \Omega}$ (counted with multiplicity).

Theorem 1.4. Suppose $\Omega$ is bounded, smooth and $B$ is constant in $\bar{\Omega}$. Given $a \in \mathbb{R}$, the following limit holds,

$$
\begin{aligned}
\lim _{h \rightarrow 0} h^{-1 / 2} \sum_{j}\left[e_{j}(h)-b h-a h^{3 / 2}\right]_{-} & = \\
& \frac{|\partial \Omega| b^{3 / 2}}{2 \pi} \int_{\mathbb{R}}\left(\mu_{1}(\xi)-1\right)_{-} d \xi+\frac{|\Omega| b}{2 \pi}[a]_{+} .
\end{aligned}
$$

Remark 1.5. The first term on the r.h.s. of (1.11) corresponds to boundary states (i.e. eigenfunctions localized near the boundary) and the second one corresponds to bulk states. Theorem 1.4 sharpens our understanding of the transition from boundary states to bulk states.

Remark 1.6. Since inf $\mathrm{Spec}_{\mathrm{ess}} P_{h, \Omega}=b h$ in the case of exterior domains, we see that the left hand side of (1.11) diverges for any $a>0$. That forces us to restrict to bounded $\Omega$. Our methods would also apply to non-constant $B$, but the order to which the bulk term appears depends on the local behavior of $B$ near the set $\{x: B(x)=b\}$. For simplicity of exposition we therefore restrict to the case of constant field.

The approach we follow is inspired by that of Lieb, Solovej and Yngvason [16], but extended with the construction of coherent states for the half-plane operator. In order to control the errors resulting from the approximations, we make use of a Lieb-Thirring inequality for magnetic operators (see [5, 16] and references therein), and a rough estimate of the energy of bound states for the case of a cylindric domain.

The paper is organized as follows. We collect in Section 2 some preliminaries. In Section 3, we determine a rough bound for the energy of bound states of the operator (1.1) in the case when the domain $\Omega$ is a cylinder. Section 4 is devoted to the construction of coherent states. In Section 5, we prove Theorem 1.1. Finally, in Section 6 we give the additional details to achieve the proof of Theorem 1.4.

\section{Preliminaries}

2.1. Lieb-Thirring inequality. Let $B \in C^{1}\left(\mathbb{R}^{2} ; \mathbb{R}\right) \cap L^{\infty}\left(\mathbb{R}^{2}\right)$ be a magnetic field such that $B(x)>0$ for all $x \in \mathbb{R}^{2}$. The vector field defined by

$$
A(x)=\frac{1}{2}\left(-\int_{0}^{1} s B(s x) x_{2} d s, \int_{0}^{1} s B(s x) x_{1} d s\right) \quad \forall x=\left(x_{1}, x_{2}\right) \in \mathbb{R}^{2},
$$

provides a magnetic potential for $B=\operatorname{curl} A$.

Consider the Schrödinger (Pauli) operator

$$
H_{\mathbb{R}^{2}}=-(\nabla-i A)^{2}-B \quad \text { in } L^{2}\left(\mathbb{R}^{2}\right) .
$$

We have the following Lieb-Thirring estimate for the negative eigenvalues of $H_{\mathbb{R}^{2}}+V$ (see [5] and references therein). 
Theorem 2.1. There exists a universal constant $C>0$ such that the following estimate is valid for the sum of the negative eigenvalues $\left\{e_{j}\right\}_{j \geq 1}$ of the operator $H=H_{\mathbb{R}^{2}}+V$,

$$
\sum_{j}\left|e_{j}\right| \leq C\left(\|B\|_{L^{\infty}\left(\mathbb{R}^{2}\right)} \int_{\mathbb{R}^{2}}[V]_{-} d x+\int_{\mathbb{R}^{2}}[V]_{-}^{2} d x\right) .
$$

2.2. Variational principles. Let $H$ be a self-adjoint operator in $L^{2}\left(\mathbb{R}^{2}\right)$ (of domain $D(H)$ ) such that

$$
(\mathrm{H}) \quad\left\{\begin{array}{l}
\inf \sigma_{\mathrm{ess}}(H) \geq 0 \\
H \mathbf{1}_{-\infty, 0}(H) \text { is trace class . }
\end{array}\right.
$$

We shall need the following two simple variational principles concerning the operator $H$, which are frequently used in [16, 5].

Lemma 2.2. Let $\gamma$ be a bounded operator such that $0 \leq \gamma \leq 1$ (in the sense of quadratic forms) and the operator $H \gamma$ is trace class. Then it holds that,

$$
\operatorname{tr}\left(H \mathbf{1}_{]-\infty, 0[}(H)\right) \leq \operatorname{tr}(H \gamma) .
$$

Lemma 2.3. Assume that the operator $H$ satisfies the hypothesis $(\mathrm{H})$. Then it holds that,

$$
\operatorname{tr}\left(H \mathbf{1}_{]-\infty, 0[}(H)\right)=\inf \sum_{j=1}^{N}\left\langle f_{j}, H f_{j}\right\rangle,
$$

where the infimum is taken over all orthonormal families $\left\{f_{1}, f_{2}, \ldots, f_{N}\right\} \subset$ $D(H)$ and $N \geq 1$.

2.3. Boundary coordinates. The closed quadratic form associated with the operator (1.1) is

$$
q_{h, \Omega}(u)=\int_{\Omega}|(h \nabla-i \mathbf{A}) u|^{2} d x
$$

with the form domain

$$
H_{h, \mathbf{A}}^{1}(\Omega)=\left\{u \in L^{2}(\Omega):(h \nabla-i \mathbf{A}) u \in L^{2}(\Omega)\right\} .
$$

The following magnetic potential generates a constant unit magnetic field,

$$
\mathbf{A}_{0}\left(x_{1}, x_{2}\right)=\left(-x_{2}, 0\right), \quad \forall\left(x_{1}, x_{2}\right) \in \mathbb{R} \times \mathbb{R} .
$$

The quadratic form,

$$
H_{h, b \mathbf{A}_{0}}^{1}\left(\mathbb{R} \times \mathbb{R}_{+}\right) \ni u \mapsto \int_{\mathbb{R} \times \mathbb{R}_{+}}\left|\left(h \nabla-i b \mathbf{A}_{0}\right) u\right|^{2} d x,
$$

with $b>0$, will serve as a model form. Actually, when the function $u \in$ $H_{h, \mathbf{A}}^{1}(\Omega)$ is supported near the boundary, the form (2.4) turns out to approximate (2.1). In order to make this precise, we introduce a convenient coordinate transformation valid in a sufficiently thin tubular neighborhood of the boundary 1. For more details on these coordinates, see for instance [12, Appendix A].

$$
\left.\Phi_{t_{0}}: \Omega\left(t_{0}\right) \ni x \mapsto(s(x), t(x)) \in \frac{|\partial \Omega|}{2 \pi} \mathbb{S}^{1} \times\right] 0, t_{0}[,
$$

\footnotetext{
${ }^{1}$ Here we assume for simplicity that $\partial \Omega$ is connected. In general, $\partial \Omega$ has finite connected components and therefore we should work on each component independently.
} 
where for $t_{0}>0, \Omega\left(t_{0}\right)$ is the tubular neighborhood of $\partial \Omega$ :

$$
\Omega\left(t_{0}\right)=\left\{x \in \Omega: \operatorname{dist}(x, \partial \Omega)<t_{0}\right\} .
$$

Let us mention that $t(x)=\operatorname{dist}(x, \partial \Omega)$ measures the distance to the boundary and $s(x)$ measures the curvilinear distance in $\partial \Omega$. We shall use the usual identification between the circle $\frac{|\partial \Omega|}{2 \pi} \mathbb{S}$ and the interval $[0,|\partial \Omega|[$. The Jacobian of the transformation $\Phi_{t_{0}}$ is equal to

$$
1-t k(s),
$$

where $k$ denotes the curvature of $\partial \Omega$.

Since $t_{0}$ will be fixed once and for all, we will sometimes omit it from the notation and simply write $\Phi$ instead of $\Phi_{t_{0}}$.

Using the coordinate transformation $\Phi_{t_{0}}$, we associate to any function $u \in L^{2}(\Omega)$, a function $\widetilde{u}$ defined in $\left[0,|\partial \Omega|\left[\times\left[0, t_{0}\right]\right.\right.$ by,

$$
\widetilde{u}(s, t)=u\left(\Phi_{t_{0}}^{-1}(s, t)\right) .
$$

Furthermore, the function $\widetilde{u}$ extends naturally to a $|\partial \Omega|$-periodic function in $s \in \mathbb{R}$.

We get then the following change of variable formulae.

Proposition 2.4. Let $u \in H_{\mathbf{A}}^{1}\left(\Omega\left(t_{0}\right)\right)$. We write $\widetilde{u}(s, t)=u\left(\Phi_{t_{0}}(s, t)\right)$,

$$
\tilde{\mathbf{A}}_{1}=\mathbf{A}_{1} \circ \Phi_{t_{0}}, \quad \tilde{\mathbf{A}}_{2}=\mathbf{A}_{2} \circ \Phi_{t_{0}} .
$$

Then we have :

$$
\begin{aligned}
\int_{\Omega\left(t_{0}\right)}|(\nabla-i \mathbf{A}) u|^{2} d x & =\int_{0}^{|\partial \Omega|} \int_{0}^{t_{0}}\left[\left|\left(\partial_{s}-i \tilde{\mathbf{A}}_{1}\right) \widetilde{u}\right|^{2}\right. \\
+ & \left.(1-t k(s))^{-2}\left|\left(\partial_{t}-i \tilde{\mathbf{A}}_{2}\right) \widetilde{u}\right|^{2}\right](1-t k(s)) d s d t
\end{aligned}
$$

and

$$
\int_{\Omega\left(t_{0}\right)}|u(x)|^{2} d x=\int_{0}^{|\partial \Omega|} \int_{0}^{t_{0}}|\widetilde{u}(s, t)|^{2}(1-t k(s)) d s d t .
$$

We will use the symbol $U_{\Phi}$ for the operator that maps $u$ to $\widetilde{u}$. We shall frequently make use of the next standard lemma, taken from [9, Lemma 3.5].

Lemma 2.5. There exists a constant $C>0$ and for all $S_{1} \in[0,|\partial \Omega|[$, $\left.S_{2} \in\right] S_{1},|\partial \Omega|\left[\right.$, there exists a function $\phi \in C_{0}^{2}\left(\left[S_{1}, S_{2}\right] \times\left[0, t_{0}\right] ; \mathbb{R}\right)$ such that, for all

$$
\left.\widetilde{S} \in\left[S_{1}, S_{2}\right], \quad \mathcal{T} \in\right] 0, t_{0}\left[, \quad \varepsilon \in\left[C \mathcal{T}, C t_{0}\right],\right.
$$

and for all $u \in H_{h, \mathbf{A}}^{1}(\Omega)$ satisfying

$$
\operatorname{supp} \widetilde{u} \subset\left[S_{1}, S_{2}\right] \times[0, \mathcal{T}],
$$

one has the following estimate,

$$
\begin{aligned}
& \left.\left|q_{h, \Omega}(u)-\int_{\mathbb{R} \times \mathbb{R}_{+}}\right|\left(h \nabla-i \widetilde{B} \mathbf{A}_{0}\right) e^{i \phi / h} \widetilde{u}\right|^{2} d s d t \mid \\
& \leq \int_{\mathbb{R}_{\mathbb{R}_{+}}}\left(\varepsilon\left|\left(h \nabla-i \widetilde{B} \mathbf{A}_{0}\right) e^{i \phi} \widetilde{u}\right|^{2}+C \varepsilon^{-1}\left(\left(S^{2}+\mathcal{T}^{2}\right)^{2}+h^{2}\right)|\widetilde{u}|^{2}\right) d s d t .
\end{aligned}
$$

Here, $S=S_{2}-S_{1}, \widetilde{B}=\widetilde{B}(\widetilde{S}, 0)$, the function $\widetilde{u}$ is associated to $u$ by (2.6) and extended by 0 on $\mathbb{R} \times \mathbb{R}_{+} \backslash \operatorname{supp} \widetilde{u}$. 
2.4. A family of one-dimensional differential operators. Let us recall the main results obtained in [4, 12] concerning the family of harmonic oscillators with Neumann boundary condition. Given $\xi \in \mathbb{R}$, we define the quadratic form,

$$
B^{1}\left(\mathbb{R}_{+}\right) \ni u \mapsto q[\xi](u)=\int_{\mathbb{R}_{+}}\left|u^{\prime}(t)\right|^{2}+|(t-\xi) u(t)|^{2} d t,
$$

where, for a positive integer $k \in \mathbb{N}$ and a given interval $I \subseteq \mathbb{R}$, the space $B^{k}(I)$ is defined by :

$$
B^{k}(I)=\left\{u \in H^{k}(I) ; \quad t^{j} u(t) \in L^{2}(I), \quad \forall j=1, \ldots, k\right\} .
$$

Since the quadratic form (2.9) is closed and symmetric it defines a unique self-adjoint operator $\mathcal{L}[\xi]$. This operator has domain,

$$
D(\mathcal{L}[\xi])=\left\{u \in B^{2}\left(\mathbb{R}_{+}\right) ; \quad u^{\prime}(0)=0\right\},
$$

and is the realization of the differential operator,

$$
\mathcal{L}[\xi]=-\partial_{t}^{2}+(t-\xi)^{2},
$$

on the given domain. We denote by $\left\{\mu_{j}(\xi)\right\}_{j=1}^{+\infty}$ the increasing sequence of eigenvalues of $\mathcal{L}[\xi]$, which are all simple. By the min-max principle, we have,

$$
\mu_{1}(\xi)=\inf _{u \in B^{1}\left(\mathbb{R}_{+}\right), u \neq 0} \frac{q[\xi](u)}{\|u\|_{L^{2}\left(\mathbb{R}_{+}\right)}^{2}} .
$$

It follows from analytic perturbation theory (see [15]) that the functions

$$
\mathbb{R} \ni \xi \mapsto \mu_{j}(\xi)
$$

are analytic.

As recalled in the introduction, $\mu_{1}(0)=1$ and $\left|\mu_{1}(\xi)-1\right|$ decays like $\exp \left(-\xi^{2}\right)$ as $\xi \rightarrow+\infty$ (see [3]) thus yielding that

$$
\int_{0}^{\infty}\left(\mu_{1}(\xi)-1\right) d \xi=-\int_{\mathbb{R}}\left[\mu_{1}(\xi)-1\right]_{-} d \xi \quad \text { is finite. }
$$

We define the constant :

$$
\Theta_{0}=\inf _{\xi \in \mathbb{R}} \mu_{1}(\xi) .
$$

Let us recall an important consequence of standard Sturm-Liouville theory (c.f.. [9, Lemma 2.1]).

Lemma 2.6. The second eigenvalue satisfies,

$$
\inf _{\xi \in \mathbb{R}} \mu_{2}(\xi)>1 .
$$

Notice that part of this conclusion is a consequence of the analysis of Dauge-Helffer [4], who show that the infimum of $\mu_{2}(\xi)$ is attained for a unique $\xi_{2} \in \mathbb{R}$. 


\section{Rough EnERgy BOUnd FOR THE CYLINDER}

Let us consider the operator (1.1) in the particular case of a cylindric domain

$$
\Omega=[0, S] \times] 0, h^{1 / 2} T[.
$$

Functions in the domain of $P_{h, \Omega}$ satisfy Neumann condition at $t=0$, periodic conditions at $s \in\{0, S\}$ and Dirichlet condition at $t=h^{1 / 2} T$. We assume in addition that the magnetic field is constant $B(x)=b, b>0$, and that the magnetic potential $A=A_{0}$ is the one given in (2.3). In this particular case, the operator has compact resolvent, hence the spectrum consists of an increasing sequence of eigenvalues $\left(e_{j}\right)_{j \geq 1}$ converging to $+\infty$. In particular, given $\lambda>0$, the energy

$$
\mathcal{E}(\lambda, b, S, T)=\sum_{j}\left[h b(1+\lambda)-e_{j}\right]_{+}
$$

is finite. Our aim in this section is to provide a rough estimate of this energy.

Lemma 3.1. There exist positive constants $T_{0}$ and $\lambda_{0}$ such that, for all $S>0, b>0, T \geq \sqrt{b} T_{0}$ and $\left.\left.\lambda \in\right] 0, \lambda_{0}\right]$, we have,

$$
\mathcal{E}(\lambda, b, S, T) \leq(1+\lambda) h b\left(\frac{S T}{2 \pi \sqrt{h}}+1\right) .
$$

Proof. By separation of variables and a scaling we may decompose $P_{h, \Omega}$ as a direct sum:

$$
\bigoplus_{n \in \mathbb{Z}} h b\left(-\frac{d^{2}}{d t^{2}}+\left(2 \pi n h^{1 / 2} b^{-1 / 2} S^{-1}+t\right)^{2}\right) \quad \text { in } \bigoplus_{n \in \mathbb{Z}} L^{2}(] 0, T / \sqrt{b}[),
$$

with Neumann boundary condition at the origin and Dirichlet condition at $t=T / \sqrt{b}$. Therefore, we may express the energy (3.1) in the form,

$$
\mathcal{E}(\lambda, b, S, T)=h b \sum_{\substack{n \in \mathbb{Z} \\ j \in \mathbb{N}}}\left[1+\lambda-\mu_{j}\left(2 \pi n h^{1 / 2} b^{-1 / 2} S^{-1} ; T / \sqrt{b}\right)\right]_{+} .
$$

Here, for a given $\xi \in \mathbb{R}$, we denote by $\mu_{j}(\xi ; \mathcal{T})$ the increasing sequence of eigenvalues for the operator

$$
-\partial_{t}^{2}+(t-\xi)^{2} \quad \text { in } L^{2}(] 0, \mathcal{T}[)
$$

with Neumann condition at the origin, and Dirichlet condition at $t=\mathcal{T}$. Notice that for a fixed $\mathcal{T}$, the min-max principle gives immediately, $\mu_{1}(\xi ; \mathcal{T}) \rightarrow$ $+\infty$ as $\xi \rightarrow+\infty$. Hence, the sum on the right hand side of (3.2) is finite. It follows also from the min-max principle that $\mu_{2}(\xi ; \mathcal{T}) \geq \mu_{2}(\xi)$, where $\mu_{2}(\xi)$ is the second eigenvalue of the operator (2.11). Now, Lemma 2.6 gives the existence of a sufficiently small $\lambda_{0}$ such that $\mu_{2}(\xi)>1+\lambda_{0}$ for all $\xi \in \mathbb{R}$. Thus, taking $\lambda \in] 0, \lambda_{0}[$,

$$
\mathcal{E}(\lambda, b, S, T)=h b \sum_{n \in \mathbb{Z}}\left[1+\lambda-\mu_{1}\left(2 \pi n h^{1 / 2} b^{-1 / 2} S^{-1} ; T / \sqrt{b}\right)\right]_{+} .
$$

By (3.3), if one can localize the set,

$$
\left\{\xi \in \mathbb{R}: \mu_{1}(\xi ; T / \sqrt{b}) \leq 1+\lambda\right\},
$$


then one gets immediately an estimate of the energy $\mathcal{E}(\lambda, b, S, T)$. Notice that for $t \leq \mathcal{T}$ and $|\xi| \geq 2 \mathcal{T}$, it holds that $(t-\xi)^{2} \geq \mathcal{T}^{2}$, hence by the minmax principle, $\mu_{1}(\xi ; \mathcal{T}) \geq \mathcal{T}^{2}$. Therefore, choosing $\mathcal{T}_{0}^{2}>1+\lambda_{0}$, it holds for $\mathcal{T} \geq \mathcal{T}_{0}$ and $\left.\lambda \in\right] 0, \lambda_{0}[$,

$$
\mu_{1}(\xi ; \mathcal{T}) \leq 1+\lambda \Longrightarrow|\xi| \leq 2 \mathcal{T}
$$

From the above localization, the estimate of Lemma 3.1 becomes a consequence of $(\underline{3.3})$.

\section{Generalized eigenprojectors}

4.1. Eigenprojectors in $\mathbb{R}^{2}$. Let $\mathbf{A}_{0}$ be the magnetic potential given in (2.3),$b>0$ and $P_{h, b, \mathbb{R}^{2}}$ the self-adjoint operator

$$
P_{h, b, \mathbb{R} \times \mathbb{R}_{+}}=-\left(h \nabla-i b \mathbf{A}_{0}\right)^{2} \quad \text { in } L^{2}\left(\mathbb{R}^{2}\right) .
$$

We recall in this section the well-known eigenprojectors for the operator $P_{h, b, \mathbb{R}^{2}}$. These are projectors $\Pi_{j}^{L}(h, b), j \geq 1$, on the Landau levels that satisfy in particular,

$$
P_{h, b, \mathbb{R}^{2}} \Pi_{j}^{L}(h, b)=(2 j-1) \Pi_{j}^{L}(h, b), \quad \sum_{j=1}^{\infty} \Pi_{j}^{L}(h, b)=\operatorname{Id}_{L^{2}\left(\mathbb{R}^{2} ; \mathbb{C}\right)} .
$$

The integral kernels (denoted again by $\Pi_{j}^{L}(h, b)$ ) are given explicitly (see [16, (3.13)]),

$$
\begin{aligned}
\Pi_{j}^{L}(h, b)(x, y) & =\frac{b}{2 \pi h} \exp \left(\frac{i b\left(y_{1} y_{2}-x_{1} x_{2}\right)}{2 h}\right) \\
& \times \exp \left(\frac{i b\left(x_{1} y_{2}-x_{2} y_{1}\right)}{2 h}-\frac{b|x-y|^{2}}{4 h}\right) L_{j-1}\left(\frac{b|x-y|^{2}}{2 h}\right),
\end{aligned}
$$

where $L_{j}$ are Laguerre polynomials normalized so that $L_{j}(0)=1$. We will need the fact that,

$$
\Pi_{j}^{L}(x, x)=\frac{b}{2 \pi h}, \quad \forall x \in \mathbb{R}^{2} .
$$

4.2. Eigenprojectors in the half-space. We construct in this section projections on the (generalized) eigenfunctions for the operator (1.1) in the case $\Omega=\mathbb{R} \times \mathbb{R}_{+}$.

Let $\mathbf{A}_{0}$ be the magnetic potential given in (2.3),$b>0$ and $P_{h, b, \mathbb{R} \times \mathbb{R}_{+}}$the Neumann realization of the operator

$$
P_{h, b, \mathbb{R} \times \mathbb{R}_{+}}=-\left(h \nabla-i b \mathbf{A}_{0}\right)^{2} \quad \text { in } L^{2}\left(\mathbb{R} \times \mathbb{R}_{+}\right) .
$$

Let us denote by $\left(u_{j}(\cdot ; \xi)\right)_{j=1}^{\infty}$ an orthonormal family of real-valued eigenfunctions of the operator $\mathcal{L}[\xi]$ from (2.11) , i.e.

$$
\left\{\begin{array}{l}
-u_{j}^{\prime \prime}(t ; \xi)+(t-\xi)^{2} u_{j}(t ; \xi)=\mu_{j}(\xi) u_{j}(t ; \xi), \quad \text { in } \mathbb{R}_{+}, \\
u_{j}^{\prime}(0 ; \xi)=0 \\
\int_{\mathbb{R}_{+}} u_{j}(t ; \xi)^{2} d t=1 .
\end{array}\right.
$$

Let us define a bounded function $\mathbb{R} \times \mathbb{R}_{+} \ni(s, t) \mapsto v_{j}(s, t ; \xi)$ by :

$$
v_{j}(s, t ; \xi)=\exp (-i \xi s) u_{j}(t ; \xi) .
$$


We introduce a family of projectors $\Pi_{j}(\xi)$ on the functions $v_{j}$,

$$
C_{0}^{\infty}\left(\overline{\mathbb{R} \times \mathbb{R}_{+}}\right) \ni \varphi \mapsto \int_{{\mathbb{R} \times \mathbb{R}_{+}}_{j}} v_{j}\left(x_{1}, x_{2} ; \xi\right) \overline{v_{j}\left(y_{1}, y_{2} ; \xi\right)} \varphi\left(y_{1}, y_{2}\right) d y_{1} d y_{2} .
$$

The projectors $\Pi_{j}$ are kernel operators. For $j \in \mathbb{N}$, the integral kernels are defined by:

$$
K_{j}(\xi)\left(\left(x_{1}, x_{2}\right),\left(y_{1}, y_{2}\right)\right)=v_{j}\left(x_{1}, x_{2} ; \xi\right) \times \overline{v_{j}\left(y_{1}, y_{2} ; \xi\right)} .
$$

Now, one easily verifies the following properties :

$$
\sum_{j=1}^{\infty} \int_{\mathbb{R}} \Pi_{j}(\xi) d \xi=2 \pi \operatorname{Id}_{L^{2}\left(\mathbb{R} \times \mathbb{R}_{+}\right)},
$$

and

$$
P_{1,1, \mathbb{R} \times \mathbb{R}_{+}} \Pi_{j}(\xi)=\mu_{j}(\xi) \Pi_{j}(\xi) .
$$

By means of a dilation, we get a family of eigenprojectors for the operator $P_{h, b, \mathbb{R} \times \mathbb{R}_{+}}$. Let us introduce the unitary operator,

$$
U_{h, b}: L^{2}\left(\mathbb{R} \times \mathbb{R}_{+}\right) \ni \varphi \mapsto U_{h, b} \varphi \in L^{2}\left(\mathbb{R} \times \mathbb{R}_{+}\right),
$$

such that, for all $x=\left(x_{1}, x_{2}\right) \in \mathbb{R} \times \mathbb{R}_{+}$,

$$
\left(U_{h, b} \varphi\right)(x)=\sqrt{b / h} \varphi(\sqrt{b / h} x) .
$$

Notice that

$$
U_{h, b}^{-1} P_{h, b, \mathbb{R} \times \mathbb{R}_{+}} U_{h, b}=h b P_{1,1, \mathbb{R} \times \mathbb{R}_{+}} .
$$

Then we introduce the family of projectors,

$$
\Pi_{j}(h, b ; \xi)=U_{h, b} \Pi_{j}(\xi) U_{h, b}^{-1} .
$$

Again, the projectors $\Pi_{j}(h, b ; \xi)$ are kernel operators. For $j \in \mathbb{N}$, the integral kernels are given via the kernels (4.4):

$$
\begin{aligned}
K_{j}(h, b ; \xi)(x, y) & =\frac{b}{h} K_{j}(\xi)(\sqrt{b / h} x, \sqrt{b / h} y) \\
& =\frac{b}{h} e^{-i \sqrt{b / h} \xi\left(x_{1}-y_{1}\right)} u_{j}\left(\sqrt{b / h} x_{2} ; \xi\right) \overline{u_{j}\left(\sqrt{b / h} y_{2} ; \xi\right)}
\end{aligned}
$$

for all $x, y \in \mathbb{R} \times \mathbb{R}_{+}$. Now, the following properties are directly inferred from (4.5) and (4.6):

$$
\sum_{j=1}^{\infty} \int_{\mathbb{R}} \Pi_{j}(h, b ; \xi) d \xi=2 \pi \operatorname{Id}_{L^{2}\left(\mathbb{R} \times \mathbb{R}_{+}\right)},
$$

and

$$
P_{h, b, \mathbb{R} \times \mathbb{R}_{+}} \Pi_{j}(h, b ; \xi)=h b \mu_{j}(\xi) \Pi_{j}(h, b ; \xi) .
$$




\section{Proof of Theorem 1.1}

5.1. Existence of discrete spectrum. Recall the operator $P_{h, \Omega}$ introduced in (1.1). The following inequality holds (see [1]) for compactly supported functions

$$
\int_{\Omega}|(h \nabla-i \mathbf{A}) \varphi|^{2} d x \geq h \int_{\Omega} B(x)|\varphi|^{2} d x, \quad \forall \varphi \in C_{0}^{\infty}(\Omega) .
$$

Using then a 'magnetic' version of Persson's Lemma (see [2, 18]), we get that

$$
\inf \operatorname{Spec}_{\mathrm{ess}} P_{h, \Omega} \geq b h,
$$

hence proving the first statement of Theorem 1.1. Let $\left\{e_{j}(h)\right\}$ be the sequence of eigenvalues (counted with multiplicity) corresponding to $\left.\sigma\left(P_{h, \Omega}\right) \cap\right]-$ $\infty, b h\left[\right.$, and define $H=P_{h, \Omega}-b h$. It suffices now to show that

$$
\sum_{j}\left\langle f_{j}, H f_{j}\right\rangle>-\infty
$$

where the functions $f_{j}$ are $L^{2}$ normalized eigenfunctions associated with the eigenvalues $e_{j}$. To that end, we introduce a partition of unity of $\mathbb{R}$,

$$
\left.\psi_{1}^{2}+\psi_{2}^{2}=1, \quad \operatorname{supp} \psi_{1} \subset\right]-\infty, 1\left[, \quad \operatorname{supp} \psi_{2} \subset\left[\frac{1}{2}, \infty[,\right.\right.
$$

and set for $k=1,2, \chi_{k}(x)=\psi_{k}(t(x)), x \in \mathbb{R}^{2}$, where $t(x)$ is the signed distance to $\partial \Omega$,

$$
t(x)=\operatorname{dist}(x, \partial \Omega) \quad \text { if } x \in \Omega, \quad t(x)=-\operatorname{dist}(x, \partial \Omega) \quad \text { otherwise. }
$$

By the IMS formula, we write,

$$
\left\langle f_{j}, H f_{j}\right\rangle=\sum_{k=1}^{2}\left(\left\langle\chi_{k} f_{j}, H \chi_{k} f_{j}\right\rangle-h^{2}\left\|\left|\nabla \chi_{k}\right| u\right\|^{2}\right),
$$

where $\|\cdot\|$ denotes the $L^{2}$ norm in $\Omega$. Using the bound $B \geq b$ together with the fact that $\psi_{1}^{2}+\psi_{2}^{2}=1$, we get a further decomposition of (5.2),

$$
\left\langle f_{j}, H f_{j}\right\rangle=\sum_{k=1}^{2}\left\langle f_{j}, \chi_{k}(H-V) \chi_{k} f_{j}\right\rangle \geq \sum_{k=1}^{2}\left\langle f_{j}, \chi_{k}\left(H_{0}-V\right) \chi_{k} f_{j}\right\rangle,
$$

where $H_{0}=-(h \nabla-i \mathbf{A})^{2}-h B$ and

$$
V=h^{2}\left(\left|\nabla \chi_{1}\right|^{2}+\left|\nabla \chi_{2}\right|^{2}\right) \text {. }
$$

Pick an arbitrary positive integer $N \leq \operatorname{Card}\left(\left\{e_{j}\right\}_{j}\right)$. Let us define the trial density matrix $L^{2}\left(\mathbb{R}^{2}\right) \ni f \mapsto \gamma_{2} f \in L^{2}\left(\mathbb{R}^{2}\right)$,

$$
\gamma_{2} f=\chi_{2} \sum_{j=1}^{N}\left\langle\chi_{2} f, f_{j}\right\rangle f_{j}
$$

which verifies the conditions $0 \leq \gamma_{2} \leq 1$ (in the sense of quadratic forms) and $\left(H_{B}-V\right) \gamma_{2}$ is trace class (actually this is a finite-rank operator). Moreover, using Lemma 2.1, we know that the operator $\left(H_{\mathbb{R}^{2}}-V\right) \mathbf{1}_{]-\infty, 0[}\left(H_{\mathbb{R}^{2}}-V\right)$ is 
trace-class, where $H_{\mathbb{R}^{2}}=-(h \nabla-i A)^{2}-h B$ is now the corresponding Pauli operator acting in $L^{2}\left(\mathbb{R}^{2}\right)$. Therefore, we deduce from Lemma 2.2 ,

$$
\begin{aligned}
\sum_{j=1}^{N}\left\langle f_{j}, \chi_{2}\left(H_{B}-V\right) \chi_{2} f_{j}\right\rangle & =\operatorname{tr}\left[\left(H_{B}-V\right) \gamma_{2}\right] \\
& \geq \operatorname{tr}\left(\left(H_{\mathbb{R}^{2}}-V\right) \mathbf{1}_{]-\infty, 0[}\left(H_{\mathbb{R}^{2}}-V\right)\right),
\end{aligned}
$$

and we notice that this bound is uniform in $N$.

To take care of the boundary contribution, we set $\omega=\operatorname{int}\left(\Omega \cap \operatorname{supp} \chi_{1}\right)$ and define the trial density matrix

$$
\gamma_{1}: L^{2}(\omega) \ni f \mapsto \gamma_{1} f=\chi_{1} \sum_{j=1}^{N}\left\langle\chi_{1} f, f_{j}\right\rangle f_{j} \in L^{2}(\omega) .
$$

Thereby, we also get,

$$
\begin{aligned}
\left\langle f_{j}, \chi_{1}\left(H_{B}-V\right) \chi_{1} f_{j}\right\rangle & =\operatorname{tr}\left[\left(H_{B}-V\right) \gamma_{1}\right] \\
& \geq \operatorname{tr}\left(\left(H_{\omega}-V\right) \mathbf{1}_{]-\infty, 0[}\left(H_{\omega}-V\right)\right),
\end{aligned}
$$

where $H_{\omega}$ is the restriction of $H_{B}$ on $\omega$ with Dirichlet condition on $\Omega \cap \partial\left(\operatorname{supp} \chi_{1}\right)$. One should notice that, since $\omega$ is bounded, $H_{\omega}-V$ has compact resolvent and hence $\left(H_{\omega}-V\right) \mathbf{1}_{]-\infty, 0[}\left(H_{\omega}-V\right)$ is evidently traceclass. Coming back to (5.2) and (5.3), we deduce that,

$$
\begin{array}{r}
-\sum_{j}\left[e_{j}(h)-b h\right]_{-}=\sum_{j}\left\langle f_{j}, H f_{j}\right\rangle \geq \\
\operatorname{tr}\left(\left(H_{\mathbb{R}^{2}}-V\right) \mathbf{1}_{]-\infty, 0[}\left(H_{\mathbb{R}^{2}}-V\right)\right) \\
+\operatorname{tr}\left(\left(H_{\omega}-V\right) \mathbf{1}_{-\infty, 0[}\left(H_{\omega}-V\right)\right),
\end{array}
$$

proving thus that the sequence $e_{j}(h)-b h$ is summable.

5.2. Lower bound. Let $\left\{f_{1}, f_{2}, \ldots, f_{N}\right\}$ now be any $L^{2}$ orthonormal set in $D(H)$. We will give a uniform lower bound to

$$
\sum_{j=1}^{N}\left\langle f_{j}, H f_{j}\right\rangle \text {. }
$$

Using Lemma 2.3, this will imply a lower bound to $\operatorname{tr}\left(H \mathbf{1}_{]-\infty, 0[}(H)\right)$.

Step 1. Localization to the boundary.

Let $\tau(h) \in] 0,1[$ be a small number to be chosen later. Using the partition of unity in (5.1), we put

$$
\psi_{1, h}(x)=\psi_{1}\left(\frac{t(x)}{\tau(h)}\right), \quad \psi_{2, h}(x)=\psi_{2}\left(\frac{t(x)}{\tau(h)}\right), \quad \forall x \in \bar{\Omega} .
$$

By the IMS formula, we write,

$$
\left\langle f_{j}, H f_{j}\right\rangle=\sum_{k=1}^{2}\left\langle f_{j}, \psi_{k, h}\left(H-V_{h}\right) \psi_{k, h} f_{j}\right\rangle,
$$

where

$$
V_{h}=h^{2}\left(\left|\nabla \psi_{1, h}\right|^{2}+\left|\nabla \psi_{2, h}\right|^{2}\right)
$$


Notice that the term corresponding to $k=2$ in (5.6) corresponds to the interior term. We will prove that it is a lower order error term. Similarly to (5.5), one can show that,

$$
\left\langle f_{j}, \psi_{1, h}\left(H-V_{h}\right) \psi_{1, h} f_{j}\right\rangle \geq \operatorname{tr}\left(\left(H_{\mathbb{R}^{2}}-V_{h}\right) \mathbf{1}_{]-\infty, 0[}\left(H_{\mathbb{R}^{2}}-V_{h}\right)\right),
$$

and we remind the reader that $H_{\mathbb{R}^{2}}=-(h \nabla-i A)^{2}-h B(x)$ is now the corresponding self-adjoint operator acting in $L^{2}\left(\mathbb{R}^{2}\right)$. Using the Lieb-Thirring inequality of Lemma 2.1, we get

$$
\begin{aligned}
& \sum_{j=1}^{N}\left\langle f_{j}, \psi_{2, h}\left(H-V_{h}\right) \psi_{2, h} f_{j}\right\rangle \\
& \quad \geq-C\left(\int_{\mathbb{R}^{2}}\left(\|B\|_{L^{\infty}}\left[-h^{-1} V_{h}\right]_{-}+\left[-h^{-1} V_{h}\right]_{-}^{2}\right) d x\right) .
\end{aligned}
$$

With our potential $V_{h}$ from (5.7), the integral on the right side above becomes of the order of $\frac{h}{\tau(h)}+\frac{h^{2}}{\tau(h)^{3}}$. Thus, we get

$$
\begin{aligned}
\sum_{j=1}^{N}\left\langle f_{j}, H f_{j}\right\rangle \geq \sum_{j=1}^{N}\left\langle f_{j}, \psi_{1, h}\left(H-V_{h}\right) \psi_{1, h} f_{j}\right\rangle & \\
& -C \frac{h}{\tau(h)}\left(1+\frac{h}{\tau(h)^{2}}\right) .
\end{aligned}
$$

Later, we shall choose $\tau(h)$ in such a manner that the first term on the right hand side above is the dominant term.

Step 2. Boundary analysis.

Here we consider the boundary term,

$$
\sum_{j=1}^{N}\left\langle f_{j}, \psi_{1, h}\left(H-V_{h}\right) \psi_{1, h} f_{j}\right\rangle .
$$

To that end, let $\chi \in L^{2}(\mathbb{R})$ be positive, smooth, supported in $] 0,1[$ and satisfying

$$
\int \chi^{2}(s) d s=1
$$

Using the boundary coordinates $(s, t)$ introduced in (2.5), we put

$$
\chi_{h}(x ; \sigma)=\frac{1}{\sqrt{\tau(h)}} \chi\left(\frac{s(x)-\sigma}{\tau(h)}\right), \quad \forall x \in \Omega\left(t_{0}\right), \forall \sigma \in \mathbb{R},
$$

and we notice that, for all $\sigma \in \mathbb{R} \backslash]-\tau(h),|\partial \Omega|$,

$$
\chi_{h}(x ; \sigma)=0 \quad \forall x \in \Omega\left(t_{0}\right) .
$$

Using again an IMS type decomposition, we write,

$$
\begin{aligned}
& \sum_{j=1}^{N}\left\langle f_{j}, \psi_{1, h}\left(H-V_{h}\right) \psi_{1, h} f_{j}\right\rangle \\
& \quad=\int_{\mathbb{R}} \sum_{j=1}^{N}\left\langle f_{j}, \psi_{1, h} \chi_{h}(x ; \sigma)\left(H-W_{h}\right) \psi_{1, h} \chi_{h}(x ; \sigma)\right\rangle d \sigma,
\end{aligned}
$$


where

$$
W_{h}=V_{h}+h^{2} \int_{\mathbb{R}}\left|\nabla \chi_{h}(x ; \sigma)\right|^{2} d \sigma .
$$

Let us denote by ( $\Phi_{t_{0}}$ is the coordinate change (2.5) valid near the boundary)

$$
u_{j, h}(\sigma)=u_{j, h}(x ; \sigma)=\psi_{1, h}(x) \chi_{h}(x ; \sigma) f_{j}(x), \quad B_{\sigma}=B\left(\Phi_{t_{0}}^{-1}(\sigma, 0)\right) .
$$

Using Lemma 2.5, we get a constant $C>0$ and a scalar function $\phi=\phi_{\sigma}$ such that, for all $C \tau(h) \leq \varepsilon \ll 1$, we have,

$$
\begin{aligned}
& \sum_{j=1}^{N}\left\langle f_{j}, \psi_{1, h} \chi_{h}(\sigma)\left(H-W_{h}\right) \psi_{1, h} \chi_{h}(\sigma)\right\rangle \geq(1-\varepsilon) \\
& \quad \times \sum_{j=1}^{N}\left\langle e^{i \phi_{\sigma} / h} \widetilde{u}_{j, h}(\sigma),\left(H_{h, B_{\sigma}, \mathbb{R} \times \mathbb{R}_{+}}-\mathcal{W}_{h}\right) e^{i \phi_{\sigma} / h} \widetilde{u}_{j, h}(\sigma)\right\rangle_{L^{2}\left(\mathbb{R} \times \mathbb{R}_{+}\right)},
\end{aligned}
$$

where

$$
H_{h, B_{\sigma}, \mathbb{R} \times \mathbb{R}_{+}}=P_{h, B_{\sigma}, \mathbb{R} \times \mathbb{R}_{+}}-b h=-\left(h \nabla-i B_{\sigma} \mathbf{A}_{0}\right)^{2}-b h,
$$

$\mathbf{A}_{0}$ is the potential introduced in (2.3),

$$
\mathcal{W}_{h}=\widetilde{W}_{h}+C \varepsilon^{-1}\left(\tau(h)^{4}+h^{2}\right),
$$

and to a function $v(x)$, we associate the function $\widetilde{v}(s, t)$ by means of (2.6). The functions $\widetilde{u}_{j, h}(\sigma)$ are naturally extended by 0 in $\mathbb{R} \times \mathbb{R}_{+} \backslash \operatorname{supp} \widetilde{u}_{j, h}(\sigma)$.

Later, we shall make a suitable choice of the parameter $\varepsilon$. We use part of the kinetic energy $H_{h, B_{\sigma}, \mathbb{R} \times \mathbb{R}_{+}}$to control the error resulting from $\mathcal{W}_{h}$. Let $\delta \in] 0,1[$ be another parameter to be specified later; $\delta$ will be chosen as a function of $h$. We decompose the previous sum in two,

$$
\begin{aligned}
& \sum_{j=1}^{N}\left\langle e^{i \phi_{\sigma} / h} \widetilde{u}_{j, h}(\sigma),\left(H_{h, B_{\sigma}, \mathbb{R} \times \mathbb{R}_{+}}-\mathcal{W}_{h}\right) e^{i \phi_{\sigma} / h} \widetilde{u}_{j, h}(\sigma)\right\rangle_{L^{2}\left(\mathbb{R} \times \mathbb{R}_{+}\right)} \\
& =(1-\delta) \sum_{j=1}^{N}\left\langle e^{i \phi_{\sigma} / h} \widetilde{u}_{j, h}(\sigma), H_{h, B_{\sigma}, \mathbb{R} \times \mathbb{R}_{+}} e^{i \phi_{\sigma} / h} \widetilde{u}_{j, h}(\sigma)\right\rangle_{L^{2}\left(\mathbb{R} \times \mathbb{R}_{+}\right)} \\
& \quad+\delta \sum_{j=1}^{N}\left\langle e^{i \phi_{\sigma} / h} \widetilde{u}_{j, h}(\sigma),\left(H_{h, B_{\sigma}, \mathbb{R} \times \mathbb{R}_{+}}-\delta^{-1} \mathcal{W}_{h}\right) e^{i \phi_{\sigma} / h} \widetilde{u}_{j, h}(\sigma)\right\rangle_{L^{2}\left(\mathbb{R} \times \mathbb{R}_{+}\right)}
\end{aligned}
$$

Let us estimate the last term on the right side. We take $T=T(h) \gg 1$, to be specified later, and we make the following choice of $\tau(h)$,

$$
\tau(h)=h^{1 / 2} T .
$$

Notice now that

$$
\left\|\delta^{-1} \mathcal{W}_{h}\right\|_{L^{\infty}\left(\mathbb{R} \times \mathbb{R}_{+}\right)} \leq C \delta^{-1}\left(\varepsilon^{-1} h^{2} T^{4}+h T^{-2}\right) \leq \lambda B_{\sigma} h,
$$

where we have defined

$$
\lambda=C b^{-1} \delta^{-1}\left(\varepsilon^{-1} h T^{4}+T^{-2}\right) .
$$


Furthermore, with $S=\tau(h)$ and the above notation, we define the operator $\widetilde{\gamma}$ on $L^{2}([0, S] \times] 0, h^{1 / 2} T[)$,

$$
\widetilde{\gamma} f=\sum_{j=1}^{N}\left\langle f, e^{i \phi_{\sigma} / h} \widetilde{u}_{j, h}(\sigma)\right\rangle_{L^{2}([0, S] \times] 0, h^{1 / 2} T[)} e^{i \phi_{\sigma} / h} \widetilde{u}_{j, h}(\sigma) .
$$

The next estimate is a simple application of Proposition 2.4,

$0 \leq\langle f, \widetilde{\gamma} f\rangle$

$$
\leq(1+C \tau(h)) \int_{\Omega} \chi_{h}(x ; \sigma)^{2} \psi_{1, h}^{2}(x)\left|\left(f \circ \Phi_{t_{0}}^{-1}\right)(x)\right|^{2} d x \leq C^{\prime} \tau(h)^{-1}\|f\|_{L^{2}}^{2} .
$$

Thus, $\frac{1}{C^{\prime} \tau(h)^{-1}} \widetilde{\gamma}$ is a density matrix. The operator $\widetilde{\gamma}$ is constructed so that we may write the term we wish to estimate in the following form,

$$
\begin{array}{r}
\sum_{j=1}^{N}\left\langle e^{i \phi_{\sigma} / h} \widetilde{u}_{j, h}(\sigma),\left(H_{h, B_{\sigma}, \mathbb{R} \times \mathbb{R}_{+}}-\delta^{-1} \mathcal{W}_{h}\right) e^{i \phi_{\sigma} / h} \widetilde{u}_{j, h}(\sigma)\right\rangle_{L^{2}\left(\mathbb{R} \times \mathbb{R}_{+}\right)} \\
=\operatorname{tr}\left[\left(H_{h, B_{\sigma}, \mathbb{R} \times \mathbb{R}_{+}}-\delta^{-1} \mathcal{W}_{h}\right) \widetilde{\gamma}\right] .
\end{array}
$$

Using the bound (5.16) on the potential $\mathcal{W}_{h}$ and the variational principle of Lemma 2.2, we get,

$$
\begin{aligned}
& \sum_{j=1}^{N}\left\langle e^{i \phi_{\sigma} / h} \widetilde{u}_{j, h}(\sigma),\left(H_{h, B_{\sigma}, \mathbb{R} \times \mathbb{R}_{+}}-\delta^{-1} \mathcal{W}_{h}\right) e^{i \phi_{\sigma} / h} \widetilde{u}_{j, h}(\sigma)\right\rangle_{L^{2}\left(\mathbb{R} \times \mathbb{R}_{+}\right)} \\
& \geq-C^{\prime} \tau(h)^{-1} \mathcal{E}\left(\lambda, B_{\sigma}, S, T\right) .
\end{aligned}
$$

Here we remind the reader that $S=\tau(h)=h^{1 / 2} T$, the energy $\mathcal{E}\left(\lambda, B_{\sigma}, S, T\right)$ is introduced in (3.1), and $C^{\prime}$ is a positive constant depending only on $\Omega$.

In order to use the estimate given in Lemma 3.1, we make the following choice of $\varepsilon$ and $\delta$,

$$
T=h^{-1 / 8}, \quad \varepsilon=h T^{6}=h^{1 / 4}, \quad \delta=T^{-3 / 2}=h^{3 / 16},
$$

so that the following conditions hold :

$$
h^{1 / 2} T \ll \varepsilon \ll 1, \quad \lambda \ll 1 .
$$

Notice that this choice implies

$$
\tau(h)=h^{3 / 8} .
$$

Using now Lemma 3.1, we conclude that (recall that $S=\tau(h)$ in our case which compensates the factor $\tau^{-1}$ from (5.18)),

$$
\begin{aligned}
& \sum_{j=1}^{N}\left\langle e^{i \phi_{\sigma} / h} \widetilde{u}_{j, h}(\sigma),\left(H_{h, B_{\sigma}, \mathbb{R} \times \mathbb{R}_{+}}-\delta^{-1} \mathcal{W}_{h}\right) e^{i \phi_{\sigma} / h} \widetilde{u}_{j, h}(\sigma)\right\rangle_{L^{2}\left(\mathbb{R} \times \mathbb{R}_{+}\right)} \\
& \quad \geq-C h^{1 / 2} T B_{\sigma}^{1 / 2}
\end{aligned}
$$

and consequently, after integration w.r.t. $\sigma \in]-\tau(h),|\partial \Omega|[$,

$$
\begin{aligned}
& \delta \int \sum_{j=1}^{N}\left\langle e^{i \phi_{\sigma} / h} \widetilde{u}_{j, h}(\sigma),\left(H_{h, B_{\sigma}, \mathbb{R} \times \mathbb{R}_{+}}-\delta^{-1} \mathcal{W}_{h}\right) e^{i \phi_{\sigma} / h} \widetilde{u}_{j, h}(\sigma\rangle_{L^{2}\left(\mathbb{R} \times \mathbb{R}_{+}\right)} d \sigma\right. \\
& \quad \geq-C \delta T h^{1 / 2}=-C h^{9 / 16}
\end{aligned}
$$


thus obtaining an error of the order $o\left(h^{1 / 2}\right)$. We point also out that with our choice, $\tau(h)=h^{3 / 8}$, so that the error in (5.8) becomes small, of the order of $h^{5 / 8}=o\left(h^{1 / 2}\right)$.

Step 3. Estimating the leading order term.

Now we estimate the first term on the right hand side of (5.15). We use the projectors $\Pi_{j}$ constructed in Section 4 . In view of (4.11) and (4.12), we have the following splitting,

$$
\begin{aligned}
H_{h, B_{\sigma}, \mathbb{R} \times \mathbb{R}_{+}} & =\frac{1}{2 \pi} \sum_{p=1}^{\infty} \int_{\mathbb{R}} H_{h, B_{\sigma}, \mathbb{R} \times \mathbb{R}_{+} \Pi_{p}\left(h, B_{\sigma} ; \xi\right) d \xi} \\
& =\frac{B_{\sigma} h}{2 \pi} \sum_{p=1}^{\infty} \int_{\mathbb{R}}\left(\mu_{p}(\xi)-\frac{b}{B_{\sigma}}\right) \Pi_{p}\left(h, B_{\sigma} ; \xi\right) d \xi
\end{aligned}
$$

Since $B_{\sigma} \geq b$, Lemma 2.6 gives that for $p \geq 2, \mu_{p}(\xi)-\frac{b}{B_{\sigma}}>0$. Hence, we get for any function $f$ in the domain of $H_{h, B_{\sigma}, \mathbb{R} \times \mathbb{R}_{+}}$,

$$
\left\langle f, H_{h, B_{\sigma}, \mathbb{R} \times \mathbb{R}_{+}} f\right\rangle \geq-\frac{B_{\sigma} h}{2 \pi} \int_{\mathbb{R}}\left[\mu_{1}(\xi)-\frac{b}{B_{\sigma}}\right]_{-}\left\langle f, \Pi_{1}\left(h, B_{\sigma} ; \xi\right) f\right\rangle d \xi .
$$

Therefore, defining

$$
\mathcal{S}_{N}(\sigma ; \xi)=\sum_{j=1}^{N}\left\langle e^{i \phi_{\sigma} / h} \widetilde{u}_{j, h}(\sigma), \Pi_{1}\left(h, B_{\sigma} ; \xi\right) e^{i \phi_{\sigma} / h} \widetilde{u}_{j, h}(\sigma)\right\rangle_{L^{2}\left(\mathbb{R} \times \mathbb{R}_{+}\right)} \geq 0
$$

we get that

$$
\begin{aligned}
& \sum_{j=1}^{N}\left\langle e^{i \phi_{\sigma} / h} \widetilde{u}_{j, h}(\sigma), H_{h, B_{\sigma}, \mathbb{R} \times \mathbb{R}_{+}} e^{i \phi_{\sigma} / h} \widetilde{u}_{j, h}(\sigma)\right\rangle_{L^{2}\left(\mathbb{R} \times \mathbb{R}_{+}\right)} \\
& \geq-\frac{B_{\sigma} h}{2 \pi} \int_{\mathbb{R}}\left[\mu_{1}(\xi)-\frac{b}{B_{\sigma}}\right]_{-} \mathcal{S}_{N}(\sigma ; \xi) d \xi
\end{aligned}
$$

We estimate now the term on the right hand side above. We start first by estimating $S_{N}(\sigma ; \xi)$. Recalling the definition of $u_{j, h}$, and using again the coordinate transformation valid near the boundary, we get (using the choice $\left.\tau(h)=h^{3 / 8}\right)$,

$$
\begin{aligned}
& \left\langle e^{i \phi_{\sigma} / h} \widetilde{u}_{j, h}(\sigma), \Pi_{1}\left(h, b_{\sigma} ; \xi\right) e^{i \phi_{\sigma} / h} \widetilde{u}_{j, h}(\sigma)\right\rangle_{L^{2}\left(\mathbb{R} \times \mathbb{R}_{+}\right)} \\
& \leq\left(1+C h^{3 / 8}\right)\left\langle f_{j}, \mathcal{P}\left(h, B_{\sigma} ; \sigma, \xi\right) f_{j}\right\rangle_{L^{2}(\Omega)}
\end{aligned}
$$

Here $\mathcal{P}\left(h, B_{\sigma} ; \sigma, \xi\right)$ is a positive operator, which is given by,

$$
\mathcal{P}\left(h, B_{\sigma} ; \sigma, \xi\right)=\psi_{1, h} \chi_{h}(\sigma) U_{\Phi}^{-1} e^{-i \phi_{\sigma} / h} \Pi_{1}\left(h, B_{\sigma} ; \xi\right) e^{i \phi_{\sigma} / h} U_{\Phi} \psi_{1, h} \chi_{h}(\sigma),
$$

and the transformation $U_{\Phi}$ is associated to the coordinate change $\Phi_{t_{0}}$ introduced in (2.5). Since $\left\{f_{1}, f_{2}, \ldots, f_{N}\right\}$ is an orthonormal family in $L^{2}(\Omega)$, we 
deduce that

$$
\begin{aligned}
\sum_{j=1}^{N}\left\langle f_{j}\right. & \left., \mathcal{P}\left(h, B_{\sigma} ; \sigma, \xi\right) f_{j}\right\rangle_{L^{2}(\Omega)} \\
\leq & \operatorname{tr}\left(\mathcal{P}\left(h, B_{\sigma} ; \sigma, \xi\right)\right) \\
= & \int_{\mathbb{R} \times \mathbb{R}_{+}} \frac{B_{\sigma}}{h}\left|\chi_{h}(s ; \sigma)\right|^{2}\left|\psi_{1, h}(t)\right|^{2}\left|u_{1}\left(h^{-1 / 2} B_{\sigma}^{1 / 2} t ; \xi\right)\right|^{2}(1-t k(s)) d s d t,
\end{aligned}
$$

where

$$
\begin{aligned}
\int_{\mathbb{R} \times \mathbb{R}_{+}}\left|\chi_{h}(s ; \sigma)\right|^{2}\left|\psi_{1, h}(t)\right|^{2}\left|u_{1}\left(h^{-1 / 2} B_{\sigma}^{1 / 2} t ; \xi\right)\right|^{2} d s d t \\
\quad \leq \tau(h)^{-1} \int_{\mathbb{R}}\left|\chi\left(\frac{s-\sigma}{\tau(h)}\right)\right|^{2} d s \int_{\mathbb{R}_{+}}\left|u_{1}\left(h^{-1 / 2} B_{\sigma}^{1 / 2} t ; \xi\right)\right|^{2} d t \\
\quad=h^{1 / 2} B_{\sigma}^{-1 / 2},
\end{aligned}
$$

and similarly,

$$
\begin{array}{rl}
\int_{\mathbb{R} \times \mathbb{R}_{+}}\left|\chi_{h}(s ; \sigma)\right|^{2}\left|\psi_{1, h}(t)\right|^{2}\left|u_{1}\left(h^{-1 / 2} B_{\sigma}^{1 / 2} t ; \xi\right)\right|^{2} & t k(s) d s d t \\
= & \mathcal{O}\left(h^{1 / 2} \tau(h)\right)=\mathcal{O}\left(h^{7 / 8}\right) .
\end{array}
$$

Coming back to (5.22) and (5.24), we get that,

$$
0 \leq S_{N}(\sigma ; \xi) \leq \frac{B_{\sigma}}{h^{1 / 2}}+\mathcal{O}\left(h^{-1 / 8}\right) .
$$

Implementing the above estimate in (5.23), we deduce the following lower bound,

$$
\begin{aligned}
& \sum_{j=1}^{N}\left\langle e^{i \phi_{\sigma} / h} \widetilde{u}_{j, h}(\sigma), H_{h, B_{\sigma}, \mathbb{R} \times \mathbb{R}_{+}} e^{i \phi_{\sigma} / h} \widetilde{u}_{j, h}(\sigma)\right\rangle_{L^{2}\left(\mathbb{R} \times \mathbb{R}_{+}\right)} \\
& \geq-\frac{h^{1 / 2} B_{\sigma}^{3 / 2}}{2 \pi} \int_{\mathbb{R}}\left[\mu_{1}(\xi)-\frac{b}{B_{\sigma}}\right]_{-} d \xi-\mathcal{O}\left(h^{7 / 8}\right),
\end{aligned}
$$

and consequently, upon integrating with respect to $\sigma$ (recall that $\widetilde{u}_{j, h}(\sigma)$ is supported in $\{0 \leq s-\sigma \leq \tau(h): s \in[0,|\partial \Omega|[\})$,

$$
\begin{array}{r}
\int \sum_{j=1}^{N}\left\langle e^{i \phi_{\sigma} / h} \widetilde{u}_{j, h}(\sigma), H_{h, B_{\sigma}, \mathbb{R} \times \mathbb{R}_{+}} e^{i \phi_{\sigma} / h} \widetilde{u}_{j, h}(\sigma)\right\rangle_{L^{2}\left(\mathbb{R} \times \mathbb{R}_{+}\right)} d \sigma \\
\geq-\int_{0}^{|\partial \Omega|} \frac{h^{1 / 2} B_{\sigma}^{3 / 2}}{2 \pi} \int_{\mathbb{R}}\left[\mu_{1}(\xi)-\frac{b}{B_{\sigma}}\right]_{-} d \xi d \sigma-\mathcal{O}\left(h^{5 / 8}\right) .
\end{array}
$$

Recalling the definition of $B_{\sigma}=B\left(\Phi_{t_{0}}^{-1}(\sigma, 0)\right)$, the integral on the r.h.s. above is actually nothing but

$$
\frac{h^{1 / 2}}{2 \pi} \int_{\partial \Omega}\left(B(x)^{3 / 2} \int_{\mathbb{R}}\left[\mu_{1}(\xi)-\frac{b}{B(x)}\right]_{-} d \xi\right) d x .
$$


Therefore, when collecting (5.8), (5.10), (5.13), (5.15), (5.21) and (5.25), we deduce finally the desired bound,

$$
\begin{aligned}
& \sum_{j=1}^{N}\left\langle f_{j}, H f_{j}\right\rangle \\
& \quad \geq-\frac{h^{1 / 2}}{2 \pi} \int_{\partial \Omega} \int_{\mathbb{R}} B(x)^{3 / 2}\left[\mu_{1}(\xi)-\frac{b}{B(x)}\right]_{-} d \xi d x-\mathcal{O}\left(h^{9 / 16}\right),
\end{aligned}
$$

uniformly with respect to $N$ and the orthonormal family $\left\{f_{j}\right\}$.

\subsection{Upper bound.}

Coherent states for the curved boundary.

Let $\sigma \in \frac{|\partial \Omega|}{2 \pi} \mathbb{S}^{1}$ (identified with $\partial \Omega$ through a parametrization of the boundary). Let $\phi=\phi_{\sigma}$ be the gauge function from Lemma 2.5. With $\Phi=\Phi_{t_{0}}$ being the coordinate change near the boundary, let $B_{\sigma}=B\left(\Phi^{-1}(\sigma, 0)\right)$ i.e. the magnetic field at the boundary point parameterized by $\sigma$. Let finally, $\chi \in C^{\infty}(\mathbb{R})$ be a positive, smooth, have supp $\chi \subset \mathbb{R}_{+}$, and be normalized such that $\int_{\mathbb{R}} \chi^{2}(s) d s=1$. Define $\chi_{h}(s)$ for $s \in[0,|\partial \Omega|[$ to be

$$
\chi_{h}(s):=\tau(h)^{-1 / 2} \chi(s / \tau(h)),
$$

and extend $\chi_{h}$ to be a $|\partial \Omega|$-periodic function. Setting

$$
\begin{aligned}
& \tilde{f}_{j}((s, t) ; h, \sigma, \xi):= \\
& \quad\left(\frac{B_{\sigma}}{h}\right)^{1 / 4} e^{-i \xi s \sqrt{B_{\sigma} / h}} u_{j}\left(\sqrt{\frac{B_{\sigma}}{h}} t ; \xi\right) e^{-i \phi_{\sigma} / h} \chi_{h}(s-\sigma) \psi_{1, h}(t),
\end{aligned}
$$

we get - by the coordinate transformation $\Phi$ - the following function in $\Omega\left(t_{0}\right)$,

$$
f_{j}(x ; h, \sigma, \xi)=\tilde{f}_{j}(\Phi(x) ; h, \sigma, \xi) .
$$

We will consider $f_{j}$ as a function on all of $\Omega$ by extension by zero to $\Omega \backslash \Omega\left(t_{0}\right)$. Let $\Pi_{j}^{\mathrm{bnd}}(h, \sigma, \xi)$ be the operator with integral kernel

$$
\Pi_{j}^{\mathrm{bnd}}(h, \sigma, \xi)\left(x, x^{\prime}\right)=f_{j}(x ; h, \sigma, \xi) \overline{f_{j}\left(x^{\prime} ; h, \sigma, \xi\right)} .
$$

In terms of the projectors constructed in (4.9), $\Pi_{j}^{\text {bnd }}(h, \sigma, \xi)$ is expressed as follows,

$$
\Pi_{j}^{\text {bnd }}(h, \sigma, \xi)=\psi_{1, h} U_{\Phi}^{-1} e^{-i \phi_{\sigma} / h} \chi_{h}(\cdot-\sigma) \Pi_{j}\left(h, B_{\sigma} ; \xi\right) e^{i \phi_{\sigma} / h} \chi_{h}(\cdot-\sigma) U_{\Phi} \psi_{1, h} .
$$

Then

$$
\begin{aligned}
\operatorname{tr} & {\left[\Pi_{j}^{\text {bnd }}(h, \sigma, \xi)\right]=\int_{\Omega}\left|f_{j}(x ; h, \sigma, \xi)\right|^{2} d x } \\
& =\iint\left|\widetilde{f}_{j}((s, t) ; \sigma, \xi)\right|^{2}(1-t k(s)) d s d t \\
& \leq\left(1+\tau(h)\|k\|_{\infty}\right) \iint \sqrt{\frac{B_{\sigma}}{h}}\left|u_{j}\left(\sqrt{\frac{B_{\sigma}}{h}} t ; \xi\right)\right|^{2} \tau(h)^{-1} \chi^{2}\left(\frac{s-\sigma}{\tau(h)}\right) d s d t \\
& =\left(1+\tau(h)\|k\|_{\infty}\right) .
\end{aligned}
$$


Also, using Lemma 2.5,

$$
\begin{aligned}
& \operatorname{tr}\left[P_{h, \Omega} \Pi_{j}^{\mathrm{bnd}}(h, \sigma, \xi)\right]=q_{h, \Omega}\left(f_{j}(\cdot ; h, \sigma, \xi)\right) \\
& \leq(1+\varepsilon) \int\left|\left(h \nabla-i B_{\sigma} \mathbf{A}_{0}\right) e^{i \phi} \tilde{f}_{j}(h, \sigma, \xi)\right|^{2} d s d t \\
& \quad+C \varepsilon^{-1}\left(\tau(h)^{4}+h^{2}\right) \int\left|\tilde{f}_{j}(h, \sigma, \xi)\right|^{2} d s d t \\
& \leq(1+\varepsilon)\left(\frac{B_{\sigma}}{h}\right)^{1 / 2} \int \chi_{h}^{2}(s-\sigma) \\
& \quad \times\left|\left(h \nabla-i B_{\sigma} \mathbf{A}_{0}\right) e^{-i \xi s \sqrt{B_{\sigma} / h}} u_{j}\left(\sqrt{\frac{B_{\sigma}}{h}} t ; \xi\right)\right|^{2} d s d t \\
& \quad+\left[(1+\varepsilon) \frac{h^{2}}{\tau(h)^{2}}+C \varepsilon^{-1}\left(\tau(h)^{4}+h^{2}\right)\right] \\
& \leq(1+\varepsilon) \mu_{1}(\xi) h B_{\sigma}+C\left[\frac{h^{2}}{\tau(h)^{2}}+\varepsilon^{-1}\left(\tau(h)^{4}+h^{2}\right)\right] .
\end{aligned}
$$

Let $M(h, \sigma, \xi, j)$ be a function with $0 \leq M \leq 1$, and write

$$
\gamma=\sum_{j=1}^{\infty} \iint M(h, \sigma, \xi, j) \Pi_{j}^{\mathrm{bnd}}(h, \sigma, \xi) \frac{d \sigma d \xi}{2 \pi \sqrt{h / B_{\sigma}}} .
$$

Then, clearly $0 \leq \gamma$ as an operator on $L^{2}(\Omega)$. We will prove that

$$
\gamma \leq\left(1+\|k\|_{\infty} \tau(h)\right) .
$$

Consider $g \in L^{2}(\Omega)$ and define $g_{\partial}=g 1_{\{\operatorname{dist}(x, \partial \Omega) \leq \tau(h)\}}$. Then

$$
\langle g \mid \gamma g\rangle=\left\langle g_{\partial} \mid \gamma g_{\partial}\right\rangle,
$$

so we may assume that $g=g_{\partial}$, i.e. that $\operatorname{supp} g \subset\{x: \operatorname{dist}(x, \partial \Omega) \leq \tau(h)\}$. We calculate,

$$
\begin{aligned}
\langle g \mid \gamma g\rangle= & \sum_{j} \iint M(h, \sigma, \xi, j) \\
& \times\left|\int \overline{\widetilde{g}(s, t)} \widetilde{f}_{j}(s, t ; h, \sigma, \xi)(1-t k(s)) d s d t\right|^{2} \frac{d \sigma d \xi}{2 \pi \sqrt{h / B_{\sigma}}} .
\end{aligned}
$$

We estimate from above by replacing $\iint M \times|\cdot|^{2}$ by $\iint 1 \times|\cdot|^{2}$ in the above expression. Then we use the fact that $u_{j}(\cdot ; \xi)$ is an orthonormal basis of $L^{2}\left(\mathbb{R}_{+}\right)$for all $\xi$. Next we evaluate the $\xi$ (Fourier) integral, which becomes

$$
\int_{-\infty}^{\infty} e^{-i \xi\left(s-s^{\prime}\right) \sqrt{B_{\sigma} / h}}=2 \pi \sqrt{h / B_{\sigma}} \delta\left(s-s^{\prime}\right) .
$$

After inserting these two results, (5.34) becomes

$$
\begin{array}{r}
\langle g \mid \gamma g\rangle \leq \int_{\sigma \in \partial \Omega} \int_{s \in \partial \Omega} \int_{t \in \mathbb{R}_{+}}|\widetilde{g}(s, t)|^{2}(1-t k(s))^{2} \\
\tau(h)^{-1} \chi^{2}\left(\frac{s-\sigma}{\tau(h)}\right) \psi_{1, h}^{2}(t) d s d t d \sigma .
\end{array}
$$


We do the $\sigma$-integration first. The normalization of $\chi$ implies that the result is

$$
\begin{aligned}
\langle g \mid \gamma g\rangle & \leq \int_{s \in \partial \Omega} \int_{t \in \mathbb{R}_{+}}|\widetilde{g}(s, t)|^{2}(1-t k(s))^{2} \psi_{1, h}^{2}(t) d s d t \\
& \leq\left(1+\tau(h)\|k\|_{\infty}\right) \int_{\Omega}|g(x)|^{2} d x .
\end{aligned}
$$

That finishes the proof of $(5.33)$.

Let $K>0$. We choose the function $M=M^{K} \cdot 1_{\{j=1\}}$, with $M^{K}$ being the characteristic function of the set

$$
\left\{(\sigma, \xi) \in \frac{|\partial \Omega|}{2 \pi} \mathbb{S}^{1} \times \mathbb{R}: \frac{b}{B_{\sigma}}-\mu_{1}(\xi) \geq 0,|\xi| \leq K\right\} .
$$

Let $\gamma^{K}$ be the corresponding density matrix. We calculate,

$$
\begin{aligned}
& \operatorname{tr}\left[\left(P_{h, \Omega}-b h\right) \gamma^{K}\right] \\
& =\iint M^{K}(h, \sigma, \xi)\left(q_{h, \Omega}\left(f_{1}(\cdot ; h, \sigma, \xi)\right)-b h\left\|f_{1}(\cdot ; h, \sigma, \xi)\right\|_{L^{2}(\Omega)}\right) \\
& \leq \iint M^{K}(h, \sigma, \xi)\left\{(1+\varepsilon) \mu_{1}(\xi) h B_{\sigma}-b h\left(1-\tau(h)\|k\|_{\infty}\right)\right. \\
& \left.\quad+C\left[\frac{h^{2}}{\tau(h)^{2}}+\varepsilon^{-1}\left(\tau(h)^{4}+h^{2}\right)\right]\right\} \frac{d \sigma d \xi}{2 \pi \sqrt{h / B_{\sigma}}} \\
& \quad+\int_{-K}^{K} \int_{0}^{|\partial \Omega|}\left[\mu_{1}(\xi) h B_{\sigma}-b h\right]_{-} \frac{d \sigma d \xi}{2 \pi \sqrt{h / B_{\sigma}}} \\
& \quad+h\left(\varepsilon\|B\|_{L^{\infty}(\partial \Omega)}+\tau(h)\|k\|_{\infty}\right)|\partial \Omega| 2 K \frac{\sqrt{\|B\|_{L^{\infty}(\partial \Omega)}}}{2 \pi \sqrt{h}} \\
& \quad+C\left[\frac{h^{2}}{\tau(h)^{2}}+\varepsilon^{-1}\left(\tau(h)^{4}+h^{2}\right)\right]|\partial \Omega| 2 K \frac{\sqrt{\|B\|_{L^{\infty}(\partial \Omega)}}}{2 \pi \sqrt{h}}
\end{aligned}
$$

The choice

$$
\tau(h)=h^{3 / 8}, \quad \varepsilon=h^{1 / 4},
$$

yields an error term which is controlled by $C K h^{3 / 4}$. Therefore,

$$
\begin{aligned}
& \operatorname{tr}\left[\left(P_{h, \Omega}-b h\right) \gamma^{K}\right] \\
& \leq-\frac{\sqrt{h}}{2 \pi} \int_{-K}^{K} \int_{0}^{|\partial \Omega|} B_{\sigma}^{3 / 2}\left[\frac{b}{B_{\sigma}}-\mu_{1}(\xi)\right]_{+} d \sigma d \xi+C K h^{3 / 4} .
\end{aligned}
$$

Since $K$ can be chosen arbitrarily large, this implies the upper bound in Theorem 1.1.

\section{Proof of Theorem 1.4}

6.1. Lower bound. We follow the strategy of the proof of Theorem 1.1. Let $H=P_{h, \Omega}-b h-a h^{3 / 2}$. We seek a uniform lower bound of

$$
\left\langle f_{j}, H f_{j}\right\rangle,
$$


for any orthonormal family $\left\{f_{1}, f_{2}, \ldots, f_{N}\right\}$ in $D(H)$. Using the notation of Section 5, we write (see (5.6)),

$$
\sum_{j=1}^{N}\left\langle f_{j}, H f_{j}\right\rangle=\sum_{k=1}^{2} \sum_{j=1}^{N}\left\langle f_{j}, \psi_{k, h}\left(H-V_{h}\right) \psi_{k, h} f_{j}\right\rangle .
$$

The term corresponding to $k=1$ is a boundary term and is estimated exactly as in Section 5 (see also (5.26)), the extra term $a h^{3 / 2}$ being included in the error term. Notice that the localization error $h^{2} \tau^{-2}=h^{5 / 4} \gg h^{3 / 2}$, so the $a h^{3 / 2}$-term can be incorporated without change to the argument. Therefore, the following lower bound holds,

$$
\begin{aligned}
\sum_{j=1}^{N}\left\langle f_{j}, \psi_{1, h}(H\right. & \left.\left.-V_{h}\right) \psi_{1, h} f_{j}\right\rangle \geq \\
& -\frac{h^{1 / 2}}{2 \pi} \int_{\partial \Omega \times \mathbb{R}} b^{3 / 2}\left[\mu_{1}(\xi)-1\right]_{-} d \xi d s(x)-\mathcal{O}\left(h^{9 / 16}\right) .
\end{aligned}
$$

Next, we estimate the interior term corresponding to $k=2$. Let us introduce a localization function $\chi \in C_{0}^{\infty}(B(0,1))$ such that $\|\chi\|_{L^{2}\left(\mathbb{R}^{2}\right)}=1$. Define

$$
\chi_{h}(x ; z)=\frac{1}{\zeta(h)} \chi\left(\frac{x-z}{\zeta(h)}\right), \quad \forall x \in \Omega, \forall z \in \mathbb{R}^{2},
$$

where $\zeta(h) \in] 0,1[$ is to be chosen later. Let us notice that

$$
\chi_{h}(x ; z)=0 \quad \forall x \in \Omega, \forall z \in \Omega+B(0, \zeta(h)) .
$$

Then we get, using the standard localization formula,

$$
\begin{aligned}
& \sum_{j=1}^{N}\left\langle f_{j}, \psi_{2, h}\left(H-V_{h}\right) \psi_{2, h} f_{j}\right\rangle \\
& \quad=\int_{\mathbb{R}^{2}} \sum_{j=1}^{N}\left\langle f_{j}, \psi_{2, h} \chi_{h}(x ; z)\left(H-W_{h}\right) \psi_{2, h} \chi_{h}(x ; z) f_{j}\right\rangle d z,
\end{aligned}
$$

where $W_{h}(x)=V_{h}(x)+h^{2} \int_{\mathbb{R}^{2}}\left|\nabla_{x} \chi(x ; z)\right|^{2} d z$.

The term corresponding to $W_{h}$ will be estimated using a Lieb-Thirring inequality. Let $\delta \in] 0,1[$ to be chosen later, and write,

$$
\begin{aligned}
& \left\langle f_{j}, \psi_{2, h} \chi_{h}(x ; z)\left(H-W_{h}\right) \psi_{2, h} \chi_{h}(x ; z) f_{j}\right\rangle \\
& =(1-\delta)\left\langle f_{j}, \varphi(x ; z) H \varphi(x ; z) f_{j}\right\rangle \\
& \quad+\delta\left\langle f_{j}, \varphi(x ; z)\left(H_{b, \mathbb{R}^{2}}-\delta^{-1} \mathcal{W}_{h}\right) \varphi(x ; z) f_{j}\right\rangle,
\end{aligned}
$$

where

$$
\begin{gathered}
\mathcal{W}_{h}(x)=\mathbf{1}_{\Omega}(x)\left(\delta a h^{3 / 2}+V_{h}(x)+h^{2} \int_{\mathbb{R}^{2}}\left|\nabla_{x} \chi(x ; z)\right|^{2} d z\right), \\
\varphi_{h}(x ; z)=\psi_{1, h}(x) \chi_{h}(x ; z)
\end{gathered}
$$

and $H_{b, \mathbb{R}^{2}}=-\left(h \nabla-i b \mathbf{A}_{0}\right)^{2}-b h$ is the self-adjoint operator acting in $L^{2}\left(\mathbb{R}^{2}\right)$. 
As explained in (5.5), the variational principle yields (i.e. Lemma 2.2),

$$
\begin{aligned}
\delta \sum_{j=1}^{N}\left\langle f_{j}, \varphi(x ; z)\left(H_{b, \mathbb{R}^{2}}-\delta^{-1} \mathcal{W}_{h}\right) \varphi(x ; z) f_{j}\right\rangle \geq \\
\quad \delta \operatorname{tr}\left(\left(H_{b, \mathbb{R}^{2}}-\delta^{-1} \mathcal{W}_{h}\right) \mathbf{1}_{]-\infty, 0[}\left(H_{b, \mathbb{R}^{2}}-\delta^{-1} \mathcal{W}_{h}\right)\right) .
\end{aligned}
$$

To estimate the last term, we use the Lieb-Thirring inequality of Lemma 2.1 . Thus we get,

$$
\begin{aligned}
\delta \sum_{j=1}^{N}\langle & \left.f_{j}, \varphi(x ; z)\left(H_{b, \mathbb{R}^{2}}-\delta^{-1} W_{h}^{-1}\right) \varphi(x ; z) f_{j}\right\rangle \\
& \geq-C \delta\left(\int_{\mathbb{R}^{2}}\left(b\left[-h^{-1} \delta^{-1} \mathcal{W}_{h}\right]_{-}+\left[-h^{-1} \delta^{-1} \mathcal{W}_{h}\right]_{-}^{2}\right) d x\right) \\
& \geq-C\left(\delta a h^{1 / 2}+\frac{h}{\tau(h)}\left(1+\delta^{-1} \frac{h}{\tau(h)^{2}}\right)+\frac{h}{\zeta(h)^{2}}\left(1+\delta^{-1} \frac{h}{\zeta(h)^{2}}\right)\right) .
\end{aligned}
$$

Recall that we have already fixed a choice of

$$
\tau(h)=h^{3 / 8} .
$$

So, for the other parameters, choosing

$$
\zeta(h)=h^{3 / 16}, \quad \delta=h^{1 / 8},
$$

we get that the term on the r.h.s. above is of order $h^{5 / 8}=o\left(h^{1 / 2}\right)$. Integrating with respect to $z \in \Omega+B(0, \zeta(h))$, we get,

$$
\begin{aligned}
\delta \int \sum_{j=1}^{N}\left\langle f_{j}, \varphi(x ; z)\left(H_{b, \mathbb{R}^{2}}-\delta^{-1} W_{h}\right) \varphi(x ; z) f_{j}\right\rangle d z & \\
& \geq-C|\Omega| h^{5 / 8}=o\left(h^{1 / 2}\right) .
\end{aligned}
$$

Now we calculate the leading order term. Recall the family of projectors $\left(\Pi_{p}^{L}\right)_{p \geq 1}$ on the Landau levels introduced in Subsection 4.1. We write,

$$
\begin{aligned}
\sum_{j=1}^{N}\left\langle f_{j},\right. & \left.\varphi(x ; z) H \varphi(x ; z) f_{j}\right\rangle \\
& =\sum_{p=1}^{\infty} \sum_{j=1}^{N}\left\langle f_{j}, \varphi(x ; z) H \Pi_{p}^{L}(h, b) \varphi(x ; z) f_{j}\right\rangle \\
& =\sum_{p=1}^{\infty} \sum_{j=1}^{N}\left\langle f_{j}, \varphi(x ; z)\left(2(p-1) b h-a h^{3 / 2}\right) \Pi_{p}^{L}(h, b) \varphi(x ; z) f_{j}\right\rangle \\
& \geq-[a]_{+} h^{3 / 2} \sum_{j=1}^{N}\left\langle f_{j}, \varphi(x ; z) \Pi_{1}^{L}(h, b) \varphi(x ; z) f_{j}\right\rangle .
\end{aligned}
$$

The operator $\varphi(x ; z) \Pi_{1}^{L}(h, b) \varphi(x ; z)$ is positive. Therefore,

$$
\sum_{j=1}^{N}\left\langle f_{j}, \varphi(x ; z) \Pi_{1}^{L}(h, b) \varphi(x ; z) f_{j}\right\rangle \leq \operatorname{tr}\left(\varphi(x ; z) \Pi_{1}^{L}(h, b) \varphi(x ; z)\right) .
$$


Using (4.1),

$$
\operatorname{tr}\left(\varphi(\cdot ; z) \Pi_{1}^{L}(h, b) \varphi(\cdot ; z)\right)=\frac{b}{2 \pi h} \int_{\Omega} \varphi^{2}(x ; z) d x \leq 1 .
$$

Hence, upon integrating w.r.t. $z \in \Omega+B(0, \zeta(h))$, we conclude the following lower bound,

$$
\begin{aligned}
(1-\delta) \sum_{j=1}^{N}\left\langle f_{j}, \varphi(x ; z) H \varphi(x ; z) f_{j}\right\rangle & \geq-\frac{h^{1 / 2} b[a]_{+}}{2 \pi}(1-\delta) \int_{\mathbb{R}^{2}} \int_{\Omega} \varphi^{2}(x ; z) d x d z \\
& \geq-\frac{h^{1 / 2} b|\Omega|}{2 \pi}[a]_{+}-\mathcal{O}\left(h^{5 / 8}\right)
\end{aligned}
$$

Combining (6.1)-(6.4), (6.5) and (6.6), we get the following lower bound,

$$
\sum_{j=1}^{N}\left\langle f_{j}, H f_{j}\right\rangle \geq-\frac{h^{1 / 2} b|\Omega|}{2 \pi}[a]_{+}-\mathcal{O}\left(h^{5 / 8}\right),
$$

which is what we desire to prove.

6.2. Upper bound. We just construct a trial density $\gamma=\gamma_{\text {int }}+\gamma_{\text {bnd }}$ and estimate $\operatorname{tr}(H \gamma)$. We take $\gamma_{\text {int }}$ to be

$$
\gamma_{\text {int }}=\psi_{2}\left(\frac{\operatorname{dist}(\cdot, \partial \Omega)}{4 h^{3 / 8}}\right) \int_{\mathbb{R}^{2}} \chi_{h}(\cdot ; z) \Pi_{1}^{L}(h, b) \chi_{h}(\cdot ; z) d z \psi_{2}\left(\frac{\operatorname{dist}(\cdot, \partial \Omega)}{4 h^{3 / 8}}\right),
$$

and $\gamma_{\text {bnd }}$ exactly as given in (5.32).

Notice that $\gamma_{\text {bnd }}$ and $\gamma_{\text {int }}$ act as direct sums since their integral kernels have disjoint support.

By calculating $\operatorname{tr}(H \gamma)=\operatorname{tr}\left(H \gamma_{\text {int }}\right)+\operatorname{tr}\left(H \gamma_{\text {bnd }}\right)$ we will get the desired upper bound. The calculation of $\operatorname{tr}\left(H \gamma_{\mathrm{bnd}}\right)$ has already been carried out in Section 5.3. In order to calculate $\operatorname{tr}\left(H \gamma_{\text {int }}\right)$, we define,

$$
\varphi(x ; z):=\psi_{2}\left(\frac{\operatorname{dist}(x, \partial \Omega)}{4 h^{3 / 8}}\right) \chi_{h}(x ; z), \quad \gamma(z)=\varphi(\cdot ; z) \Pi_{1}^{L}(h, b) \varphi(\cdot ; z) .
$$

Then,

$$
\operatorname{tr}\left(H \gamma_{\text {int }}\right)=\int \operatorname{tr}(H \gamma(z)) d z
$$

Since $\Pi_{1}^{L}$ is a projector, it follows that,

$$
\begin{aligned}
\operatorname{tr}(H \gamma(z)) & =\operatorname{tr}\left(H \varphi(x ; z) \Pi_{1}^{L}(h, b) \varphi(x ; z)\right) \\
& =\operatorname{tr}\left(\Pi_{1}^{L}(h, b) \varphi(x ; z) H \varphi(x ; z) \Pi_{1}^{L}(h, b)\right) .
\end{aligned}
$$

Using the following localization formula:

$$
\begin{aligned}
\langle H \chi f, \chi f\rangle=\operatorname{Re}\langle\chi H f, \chi f\rangle+h^{2} \||\nabla \chi|^{2} f & \|^{2}, \\
& \forall \chi \in C_{0}^{\infty}(\Omega), \forall f \in D(H),
\end{aligned}
$$

we deduce that

$$
\begin{aligned}
& \operatorname{tr}\left(\Pi_{1}^{L}(h, b) \varphi(x ; z) H \varphi(x ; z) \Pi_{1}^{L}(h, b)\right)= \\
& \quad \operatorname{Re}\left[\operatorname{tr}\left(\Pi_{1}^{L}(h, b) \varphi(x ; z)^{2} H \Pi_{1}^{L}(h, b)\right)\right]+\operatorname{tr}\left(\Pi_{1}^{L}(h, b) V_{h}(x ; z) \Pi_{1}^{L}(h, b)\right),
\end{aligned}
$$


where

$$
V_{h}(x ; z)=h^{2}|\nabla \varphi(x ; z)|^{2} .
$$

Using that the trace is cyclic, we get

$$
\begin{aligned}
& \operatorname{tr}\left(\Pi_{1}^{L}(h, b) \varphi(x ; z)^{2} H \Pi_{1}^{L}(h, b)\right)=\operatorname{tr}\left(\varphi(x ; z) H \Pi_{1}^{L}(h, b) \varphi(x ; z)\right), \\
& \operatorname{tr}\left(\Pi_{1}^{L}(h, b) V_{h}(x ; z) \Pi_{1}^{L}(h, b)\right)=\operatorname{tr}\left(\sqrt{V_{h}} \Pi_{1}^{L}(h, b) \sqrt{V_{h}}\right) .
\end{aligned}
$$

Now $H \Pi_{1}^{L}(h, b)=-a h^{3 / 2} \Pi_{1}^{L}(h, b)$. Therefore, we get,

$$
\begin{aligned}
& \operatorname{tr}\left(\varphi(x ; z) \Pi_{1}^{L}(h, b) H \Pi_{1}^{L}(h, b) \varphi(x ; z)\right) \\
& \quad=-a h^{3 / 2} \operatorname{tr}\left(\varphi(x ; z) \Pi_{1}^{L}(h, b) \varphi(x ; z)\right) .
\end{aligned}
$$

Notice now that, (recall that $\varphi(x ; z) \Pi_{1}^{L}(h, b) \varphi(x ; z)$ is a kernel operator),

$$
\begin{aligned}
\int_{\mathbb{R}^{2}} \operatorname{tr}\left(\varphi(x ; z) \Pi_{1}^{L}(h, b) \varphi(x ; z)\right) d z & =\frac{b}{2 \pi h} \int_{\Omega}\left|\psi_{2}\left(\frac{\operatorname{dist}(x, \partial \Omega)}{4 h^{3 / 8}}\right)\right|^{2} d x \\
= & \frac{b}{2 \pi h} \int_{\Omega}\left(1-\left|\psi_{1}\left(\frac{\operatorname{dist}(x, \partial \Omega)}{4 h^{3 / 8}}\right)\right|^{2}\right) d x \\
& =\frac{b}{2 \pi h}\left(|\Omega|-\int_{\Omega}\left|\psi_{1}\left(\frac{\operatorname{dist}(x, \partial \Omega)}{4 h^{3 / 8}}\right)\right|^{2} d x\right) .
\end{aligned}
$$

The function $\psi_{1}\left(\frac{\operatorname{dist}(x, \partial \Omega)}{4 h^{3 / 8}}\right)$ being supported in $\Omega\left(4 h^{3 / 8}\right)$, its $L^{2}$ integral becomes small of the order $\mathcal{O}\left(h^{3 / 8}\right)$. Therefore, coming back to (6.8), we get finally,

$$
\int_{\mathbb{R}^{2}} \operatorname{tr}\left(\varphi(x ; z) \Pi_{1}^{L}(h, b) H \Pi_{1}^{L}(h, b) \varphi(x ; z)\right) d z=-\frac{a b|\Omega|}{2 \pi} h^{1 / 2}+\mathcal{O}\left(h^{5 / 8}\right) .
$$

We need next to estimate the trace (6.9). Actually,

$$
\begin{aligned}
\operatorname{tr}\left(\sqrt{V_{h}}\right. & \left.\Pi_{1}^{L}(h, b) \sqrt{V_{h}}\right) \\
& =\frac{b}{2 \pi h} \int_{\Omega} V_{h}(x) d x \\
& \leq \frac{b h}{\pi} \int_{\Omega}\left(\left|\nabla \chi_{h}\right|^{2}+\left|\nabla \psi_{2}\left(\frac{\operatorname{dist}(x, \partial \Omega)}{4 h^{3 / 8}}\right)\right|^{2}\right) d x \\
& \leq C \frac{b h}{\pi}\left(\zeta(h)^{-2}+h^{-3 / 8}\right) .
\end{aligned}
$$

Choosing $\zeta(h)=h^{3 / 16}$ then coming back to (6.9), we get,

$$
\operatorname{tr}\left(\Pi_{1}^{L}(h, b) V_{h}(x ; z) \Pi_{1}^{L}(h, b)\right)=\mathcal{O}\left(h^{5 / 8}\right) .
$$


We integrate (6.7) w.r.t. $z \in \Omega+B(0, \zeta(h))$ and we substitute (6.10) and (6.11) in the resulting formula to get,

$$
\begin{aligned}
\operatorname{tr}\left(H \gamma_{\text {int }}\right)=\int \operatorname{tr}\left(\Pi_{1}^{L}(h, b) \varphi(x ; z) H \varphi(x ; z) \Pi_{1}^{L}(h, b)\right) d z & \\
& =-\frac{a b|\Omega|}{2 \pi} h^{1 / 2}+\mathcal{O}\left(h^{5 / 8}\right) .
\end{aligned}
$$

\section{ACKNOWLEDGEMENTS}

The authors were supported by a Starting Independent Researcher grant by the ERC under the FP7. SF is also supported by the Danish Research Council and the Lundbeck Foundation.

\section{REFERENCES}

[1] J. Avron, I. Herbst, B. Simon. Schrödinger operators with magnetic fields. I. General interactions. Duke Math. J. 45 (1978), no. 4, 847-883.

[2] V. Bonnaillie. On the fundamental state energy for a Schrödinger operator with magnetic field in domains with corners. Asymptot. Anal. 41 no. 3-4, 215-258 (2005).

[3] C. Bolley, B. Helffer. An application of semi-classical analysis to the asymptotic study of the supercooling field of a superconducting material. Ann. Inst. H. Poincaré Phys. Théor. 58 (1993), no. 2, 189-233.

[4] M. Dauge, B. Helffer. Eigenvalues variation I, Neumann problem for Sturm-Liouville operators, Journal of Differential Equations, 104 (2) 243-262 (1993).

[5] L. Erdös, J.P Solovej. Semiclassical eigenvalue estimates for the Pauli operator with strong non-homogeneous magnetic fields. II. Leading order asymptotic estimates. Comm. Math. Phys. 188 (1997).

[6] W.D. Evans, R.T. Lewis, H. Siedentop, J.P. Solovej. Counting eigenvalues using coherent states with an application to Dirac and Schrödinger operators in the semiclassical limit, Arkiv för Matematik 34 (2) 265-283 (1996).

[7] S. Fournais, B. Helffer. Accurate eigenvalue asymptotics for the magnetic Neumann Laplacian. Ann. Inst. Fourier. 56 (1) 1-67 (2006).

[8] S. Fournais, B. Helffer. On the Ginzburg-Landau critical field in three dimensions. Comm. Pure Appl. Math. (In press).

[9] R. Frank. On the asymptotic number of edge states for magnetic Schrödinger operators. Proc. London Math. Soc. (3) 95 (1) 1-19 (2007).

[10] M. Melgaard, G. Rozenblum. Eigenvalue asymptotics for weakly perturbed Dirac and Schrödinger operators with constant magnetic fields of full rank. Comm. Partial Differential Equations 28 (2003), no. 3-4, 697-736.

[11] B. Helffer, A. Morame. Magnetic bottles for the Neumann problem: curvature effects in the case of dimension 3 (general case). Ann. Sci. Ecole Norm. Sup. (4) 37 (1) 105-170 (2004).

[12] B. Helffer, A. Morame. Magnetic bottles in connection with superconductivity. J. Func. Anal. 181 (2) 604-680 (2001).

[13] A. Kachmar. On the ground state energy for a magnetic Schrödinger operator and the effect of the De Gennes boundary condition. J. Math. Phys. 47 (7) 072106 (32 pp.) 2006 .

[14] A. Kachmar. Weyl asymptotics for magnetic Schrödinger operators and de Gennes' boundary condition. Rev. Math. Phys. 20 No. 8 (2008), 901-932.

[15] T. Kato. Perturbation theory for linear operators. Springer-Verlag, Berlin, 1995.

[16] E.H. Lieb, J.P. Solovej, J. Yngvason. Asymptotics of heavy atoms in high magnetic fields. II. Semiclassical regions. Comm. Math. Phys. 161 (1994) (1) 77-124.

[17] X.-B. Pan. Analogies between superconductors and liquid crystals: nucleation and critical fields. Asymptotic analysis and singularities-elliptic and parabolic PDEs and related problems, 479-518, Adv. Stud. Pure Math., 47-2, Math. Soc. Japan, Tokyo, 2007. 
[18] A. Persson. Bounds for the discrete part of the spectrum of a semi-bounded Schrödinger operator. Math. Scand. 8 143-153 (1960).

[19] M. Persson. Eigenvalue asymptotics of the even-dimensional exterior LandauNeumann Hamiltonian. Preprint.

[20] G. Raikov, S. Warzel. Spectral asymptotics for magnetic Schrödinger operators with rapidly decreasing electric potentials. C. R. Math. Acad. Sci. Paris 335 (2002), no. 8, 683-688.

[21] M. Reed, B. Simon. Methods of Modern Mathematical Physics VI: Analysis of Operators. Academic Press, New York, 1979.

(S. Fournais and A. Kachmar) Department of Mathematical Sciences, UniverSity of Aarhus, Ny Munkegade, Building 1530, DK-8000 Århus C, Denmark

E-mail address, S. Fournais: fournais@imf.au.dk

E-mail address, A. Kachmar: ayman.kachmar@math.u-psud.fr 